\title{
ON THE RIEMANN HYPOTHESIS FOR THE ZETA FUNCTION
}

\author{
XIAO-JUN YANG
}

ABSTRACT. In this paper we address some variants for the products of Hadamard and Patterson. We prove that all zeros of the Riemann $\Xi$-function are real. We also prove that the Riemann hypothesis is true. The equivalence theorems associated with the Riemann zeta-function are obtained in detail.

\section{Contents}

1. Introduction 2

1.1. Functions 2

1.2. Analytic continuations 3

1.3. Functional equations 5

1.4. Critical trip 6

1.5. Critical line 6

1.6. Riemann-von Mangoldt formula 6

1.7. Riemann-Siegel formula 6

1.8. Riemann hypothesis 7

1.9. The target of this paper 8

1.10. Acknowledgements 8

2. Theory of the entire Riemann zeta-function 9

2.1. The integral representation 9

2.2. The series representation 9

2.3. The product representations 9

2.4. Order, exponent of convergence, and genus 14

2.5. Some equivalent classes $\quad 15$

3. Theory of the Riemann $\Xi$ function 16

3.1. Order, convergence exponent, and genus 16

3.2. Integral representations 17

3.3. Series representations 18

3.4. Product formulas 20

1991 Mathematics Subject Classification. Primary: 11-04; Secondary: 11M06, 11M26.

Key words and phrases. Riemann zeta-function, entire Riemann zeta-function, completed Riemann zeta-function, Riemann $\Xi$-function, Riemann hypothesis. 
RIEMANN HYPOTHESIS

3.5. Well-known results 23

3.6. All zeros are real 24

3.7. A detailed proof of Conjecture 1 26

3.8. Turán inequalities 27

3.9. Proofs of Lemmas 12 and $13 \quad 28$

3.10. A equivalent class 28

4. Comments 29

4.1. Nontrivial zeros 29

4.2. Some equivalence theorems 29

$\begin{array}{ll}\text { References } & 38\end{array}$

\section{INTRODUCTION}

In 1774, Euler [1] introduced the zeta-function by the real series

$$
\zeta(s)=\sum_{n=1}^{\infty} m^{-s},
$$

where $s \in \mathbb{R}, n \in \mathbb{N}$ and $s>1$, and proposed his product by

$$
\zeta(s)=\prod_{p}\left(1-p^{-s}\right)^{-1},
$$

where $p$ run through all primes and $s>1$, if we denote the sets of real and integer numbers by $\mathbb{R}$ and $\mathbb{N}$, respectively.

1.1. Functions. In his remarkable paper published in 1859, Riemann [2] extended (1) to be meromorphic continuation to the whole complex plane $s \in \mathbb{C}$ except for the simple pole $s=1$ with residue $\operatorname{Res}_{s=1}(\zeta(s))=1$ and proposed that (1) and (2) are valid for $\operatorname{Re}(s)>1$ if $\mathbb{C}$ is denoted as the set of complex numbers, and $\operatorname{Re}(s)$ and $\operatorname{Im}(s)$ are denoted as the real and imaginary part of the complex variable $s \in \mathbb{C}$. It has been proved that for $s \in \mathbb{C} \backslash\{1\}$, Eq. (1), which is well-known Riemann zeta-function, has the trivial zeros $s=-2 m$ with $m \in \mathbb{N}$ [3].

Riemann suggested the completed Riemann zeta-function by the expression [2]

$$
\varsigma(s)=\pi^{-s / 2} \Gamma(s / 2) \zeta(s),
$$

where $s \in \mathbb{C}$ and $\Gamma$ is the gamma function [4]. In his book [5], Neukirch discovered that (3) is meromorphic continuation to the whole complex plane $s \in \mathbb{C}$ except for two poles $s=1$ and $s=0$ with residues $\operatorname{Res}_{s=1}(\varsigma(s))=1$ and $\operatorname{Res}_{s=0}(\varsigma(s))=-1$, respectively. 
To remove the poles and trivial zeros of them, Riemann discovered the entire Riemann zeta-function, defined by [2]

$$
\xi(s)=\frac{1}{2} s(s-1) \pi^{-s / 2} \Gamma(s / 2) \zeta(s)
$$

for $s \in \mathbb{C}$.

In order to study the zeros of (4), Riemann defined the entire Riemann zetafunction at the critical line $\operatorname{Re}(s)=1 / 2$ by [2]

$$
\Xi(t)=\xi(1 / 2+i t),
$$

which is denoted by Landau [6], and called the Riemann $\Xi$ function for $t \in \mathbb{C}$ and $i=\sqrt{-1}$ by Pólya [7], and discovered by Riemann [2]

$$
\log \Xi(t)=\sum_{n=1}^{\infty}\left(1-\frac{t^{2}}{\tau_{n}^{2}}\right)+\log \Xi(0),
$$

where $\tau_{n}$ run the positive roots of $\Xi(t)=0$.

1.2. Analytic continuations. By (4), Riemann discovered that [2]

$$
\xi(s)=4 \int_{1}^{\infty} \frac{d\left(x^{\frac{3}{2}} \psi^{(1)}(t)\right)}{d t} t^{-\frac{1}{4}} \cosh \left(\frac{\left(s-\frac{1}{2}\right) \log t}{2}\right) d t
$$

for $s \in \mathbb{C}$ and

$$
\psi(x)=\sum_{n=1}^{\infty} e^{-n^{2} \pi x},
$$

with $x>0$.

To further study the zeros of (1) for $s \in \mathbb{C}$, Riemann showed [2]

$$
\Xi(t)=\frac{1}{2}-\left(t^{2}+\frac{1}{4}\right) \int_{1}^{\infty} \psi(x) x^{-\frac{3}{4}} \cos \left(\frac{t}{2} \log x\right) d x,
$$

or, alternatively,

$$
\Xi(t)=4 \int_{1}^{\infty} \frac{d\left(x^{\frac{3}{2}} \psi^{(1)}(x)\right)}{d x} x^{-\frac{1}{4}} \cos \left(\frac{t}{2} \log x\right) d x .
$$


By (9), Hadamard [8] in 1893 set up the theory of the entire function to deal with the series of (5), and suggested that

$$
\Xi(t)=\frac{1}{2}-\left(t^{2}+\frac{1}{4}\right) \int_{1}^{\infty} \psi(x) x^{-\frac{3}{4}} \cos \left(\frac{t}{2} \log x\right) d x=\sum_{k=0}^{\infty}(-1)^{k} \varphi_{k} t^{2 m},
$$

where

$$
\varphi_{k}=\left(\frac{D_{2 k}}{4}-D_{2 k-2}\right)
$$

and

$$
D_{k}=\frac{1}{2^{2 k} \Gamma(2 k+1)} \int_{1}^{\infty} \psi(x) x^{-\frac{3}{4}}(\log x)^{k} d x .
$$

In this case, (5) is an even entire function.

By (10), Pólya [7] in 1927 showed that

$$
\varphi_{k}=\frac{(-1)^{k} \Xi^{(2 k)}(0)}{\Gamma(2 k+1)}
$$

To find that the zeros of (5) are real, Hardy in 1914 [9] proposed the well-known result is that (5) has infinitely many zeros, proved by Hardy and Littlewood in 1921 [10]. On the other hand, the necessary condition for the real zeros of (5) is that the coefficients $\varphi_{k}$ satisfy the Turán inequalities, which was suggested in $[11,12]$. Pólya and Schur [13] considered that the product and series for (5) are equivalent to each others. They have played the important roles in the analysis of the zeta function because it is connected with the Laguerre-Pólya class in theory of the entire function [14].

In 1893, Hadamard developed [8] a well-known product for the entire Riemannzeta function by the expression [3]

$$
\xi(s)=\xi(0) \prod_{n=1}^{\infty}\left(1-\frac{s}{s_{n}}\right),
$$

where $s_{n}$ run the roots of $\xi(s)=0$ and $s \in \mathbb{C}$.

After one year, Cahen [15] discovered that

$$
\Xi(t)=\Xi(0) \prod_{n=1}^{\infty}\left(1-\frac{t^{2}}{\tau_{n}^{2}}\right)
$$

which can be deduced by (6) and was rediscovered by Titchmarsh [16], where $\tau_{n}$ run the positive roots of $\Xi(t)=0$. 
In 1974, Edwards proved that [3]

$$
\xi(s)=A_{1} \prod_{n=1}^{\infty}\left(1-\frac{s-\frac{1}{2}}{s_{n}-\frac{1}{2}}\right)
$$

and

$$
\Xi(t)=A_{2} \prod_{n=1}^{\infty}\left(1-\frac{t}{t_{n}}\right)
$$

where $t_{n}$ run the roots of $\Xi(t)=0$, and both $A_{1}$ and $A_{2}$ are the constants.

In 1988, Patterson ([17], p.34) discovered an alternative product by

$$
\xi(s)=\frac{1}{2} \prod_{n=1}^{\infty}\left(1-\frac{s}{\widehat{s_{n}}}\right)\left(1-\frac{s}{1-\widehat{s_{n}}}\right)
$$

where $s \in \mathbb{C}$ and

$$
\widehat{s_{n}}=R e\left(s_{n}\right)+i \tau_{n}
$$

1.3. Functional equations. In 1859, Riemann showed that [2]

$$
\zeta(s)=\Gamma(1-s)(2 \pi)^{s-1} 2 \sin (s \pi / 2) \zeta(1-s),
$$

where $s \in \mathbb{C}$.

For $s \in \mathbb{C}$ Landau in 1918 [18] proposed an alternative form of (21), given by

$$
\zeta(1-s)=2(2 \pi)^{-s} \cos (\pi s / 2) \Gamma(s) \zeta(s),
$$

and discovered that [18]

$$
\begin{gathered}
\varsigma(s)=\varsigma(1-s), \\
\xi(s)=\xi(1-s)
\end{gathered}
$$

and

$$
\Xi(t)=\Xi(1-t)
$$

where $s \in \mathbb{C}$.

It was reported in 1768 that Euler [19] discovered (21) and (21) in the real case. In his book (for the details, see [6], p.288), Landau also suggested that

$$
\xi(s)=\xi(1-s)=\xi\left(\frac{1}{2}+i t\right)=\xi\left(\frac{1}{2}-i t\right)=\Xi(t)=\Xi(-t),
$$

where $t \in \mathbb{R}$ and $s \in \mathbb{C}$. 
1.4. Critical trip. The critical trip for the Riemann zeta-function is

$$
0<\operatorname{Re}(s)<1
$$

which was proposed in 1859 by Riemann [2] and proved in 1896 by Hadamard [20] and Vallee-Poussin [21]. This was connected with the number of the nontrivial zeros of the Riemann zeta-function in critical trip $0<R e(s)<1$ and in the trip $0<t<T$, which is defined by $[22,23]$

$$
\widetilde{N}(T)=\#\{s=\sigma+i t: 0 \leq t \leq T, 0<\sigma<1, \zeta(s)=0\} .
$$

The completed and entire Riemann zeta-functions also have the same critical trip $0<\operatorname{Re}(s)<1[3]$.

1.5. Critical line. Riemann proposed that the critical line for the Riemann zetafunction is that all nontrivial zeros of the Riemann zeta-function lie on the line $\operatorname{Re}(s)=1 / 2$, which is also the critical line of the completed and entire Riemann zeta-functions [24, 25].

1.6. Riemann-von Mangoldt formula. The number of the nontrivial zeros for the Riemann zeta-function at the critical line and in the trip $0<t<T$ is equal to the number of the zeros for $\Xi(t)$ in the trip $0<t<T$, and we denote it by $[2,22,23]$

$$
\widetilde{N}_{0}(T)=\#\{s=1 / 2+i t: 0 \leq t \leq T, \zeta(s)=0\} .
$$

Riemann reported that $[2,22]$

$$
\widetilde{N}_{0}(T) \approx \frac{T}{2 \pi} \log \frac{T}{2 \pi e}
$$

and von Mangoldt proved in 1905 that [26, 23]

$$
\widetilde{N}_{0}(T)=\frac{T}{2 \pi} \log \frac{T}{2 \pi e}+O(\log T) .
$$

Eq. (31) is the Riemann-von Mangoldt formula due to them.

1.7. Riemann-Siegel formula. In the Riemann's Nachlass, Riemann proposed the formula of the method to find the imaginary part of the complex variable $s_{n}$, where $s_{n}$ run the roots of $\xi(s)=0$ and $s \in \mathbb{C}$. In fact, Riemann obtained few values for the imaginary part of the complex variable $s_{n}$ [2]. Based on the work of Hardy and Littlewood [10], Siegel in 1932 revised the Riemann result and obtained [27, 28]

$$
e^{i \mathbb{A}(t)} \zeta\left(\frac{1}{2}+i t\right)=2 \sum_{j=1}^{g(t)} \frac{1}{\sqrt{j}} \cos (\mathbb{A}(t)-t \log j)+\mathbb{S}(t),
$$


where $t>0$,

$$
\begin{gathered}
g(t)=\left[\sqrt{\frac{t}{2 \pi}}\right], \\
\mathbb{A}(t)=-\frac{t}{2} \log \pi+\arg \Gamma\left(\frac{1}{4}+\frac{i t}{2}\right)
\end{gathered}
$$

and [10]

$$
\mathbb{S}(t)=\mathrm{O}(1 / \sqrt[4]{t})
$$

It is clear that Riemann and Siegel [27, 28] showed that

$$
\operatorname{Im}\left(s_{n}\right)=\tau_{n}\left(\tau_{n}>0\right)
$$

subject to the condition, which implies that either [22]

$$
\widetilde{N}(T)=\widetilde{N}_{0}(T) \text {, }
$$

or [27]

$$
\operatorname{Im}\left(s_{n}\right)=t_{n}\left(t_{n}>0\right),
$$

where $\Xi(t)=0$. Different from the techniques [29], a fast algorithm for finding the nontrivial zeros for the Riemann zeta-function was developed by Odlyzko and Schönhage [30].

It is well known that (38) might be obtained by the theorem of Hardy [9] and the Turán inequalities [31], and also deduced by the product for the Riemann $\Xi$ function [3], which is an analogue of the Euler product of the cosine [32]. Here, $t_{n}$ are called the Riemann-Siegel zeros, which are well-known constants in the present paper.

1.8. Riemann hypothesis. In order to present the distribution of the prime, Riemann in 1895 [2] conjectured well-known Riemann hypothesis, which gives the assert as follows $[2,22,23]$ :

Conjecture 1. The nontrivial zeros of $\zeta(s)$ have real part equal to $1 / 2$, i.e., $\operatorname{Re}(s)=$ $1 / 2$.

This famous hypothesis remains the most important unsolved problem in theory of the analytic number theory for more than 160 years [33, 34, 35]. As a matter of fact, Riemann [2] showed that the zeros of the completed [5] and entire [36] Riemann zeta-function are the same as those of the Riemann zeta-function. This implies that the real part of the zeros of the completed [5] and entire [36] Riemann zeta-functions functions is the same as that of the Riemann zeta-function [2]. For the details, see [2, $22,23]$. The consequence of its truth implies that Lindelöf conjecture ([17], p.34), 
Montgomery pair correlation conjecture [38, 39] and the Berry-Keating conjecture in quantum chaos $[40,41]$ are true and is also related to the Yang-Lee theorem [42, 43]. For the more equivalences of this conjecture, see [44].

1.9. The target of this paper. To prove Conjecture 1, our main aim is to find a best way to provide new products for the Riemann $\Xi$-function with the nontrivial zeros $s_{n}$ of the Riemann zeta-function by the variants of the Hadamard [8] and Patterson ([17], p.34) products at the critical line because by the result coming from the theorem of Hardy [9], it is known that it may has infinitely many real zeros, which is the same as the fact that the Jensen polynomials for the Riemann zetafunction have real zeros [45]. With the assistance of the Riemann-Siegel zeros $t_{n}$, we obtain the real part of the (nontrivial) zeros for the entire Riemann zeta-function (4), which is derived from the fact, which, so-called conjecture of Jensen [7, 35, 45] proved in our paper, that all zeros for the Riemann $\Xi$-function (5) are real. This real part implies the truth of Conjecture 1.

The structure of the paper is designed as follows. In Section 2 we investigate the integral, series and product for the entire Riemann zeta- function. In Section 3 we study integral, series and product for the Riemann $\Xi$ function and give the existence theorem of the zeros for the Riemann $\Xi$-function, and prove the Riemann hypothesis. In Section 4 we mainly give the equivalence theorems.

1.10. Acknowledgements. I work on the plan of the Riemann conjecture from autumn 2018. At first, I would like to express thanks for Professor Nouzha El Yacoubi (President of African Mathematical Union), Professor Mahmoud Abdel-Aty (Vice-President of African Academy of Sciences and Sohag University), Professor Jeffrey Hoffstein (Brown University), Professor Mircea Merca (University of Craiova and Academy of Romanian Scientists), Professor J. A. Tenreiro Machado (Instituto Superior de Engenharia do Porto), Professor Mustafa Bayram (Biruni University), Professor Sohail Nadeem (Quaid-i-Azam University), Professor Miguel A. F. Sanjuan (King Juan Carlos University), Professor Minvydas Ragulskis (Kaunas University of Technology), Professor Hossein Jafari (University of South Africa), and Professor Dumitru Baleanu (Cankaya University), for supporting the plan of the Riemann conjecture from spring 2019. I would also like to thank Professor Mahouton Norbert Hounkonnou (University of Abomey-Calavi) and Professor Carlo Cattani (Tuscia University) for giving the help to improve the manuscript and discuss the Riemannzeta function. I would like to remember Professor He-Ping Xie (Shenzhen University) and Professor Feng Gao (China University of Mining and Technology) who support me to study mathematics. This work is supported by the Yue-Qi Scholar of the China University of Mining and Technology (No. 102504180004). 
RIEMANN HYPOTHESIS

\section{TheOry of the EntiRe Riemann Zeta-FunCtion}

In this section we investigate the theory of the entire Riemann zeta-function involving the integral, series and product formulas.

2.1. The integral representation. The integral representation of the entire Riemann zeta-function was first given by Riemann [2].

Lemma 1. (Riemann integral representation theorem)

Let $s \in \mathbb{C}$. If $\psi(x)$ is defined in (8), then

$$
\xi(s)=4 \int_{1}^{\infty} \frac{d\left(x^{\frac{3}{2}} \psi^{(1)}(x)\right)}{d x} x^{-\frac{1}{4}} \cosh \left(\frac{(s-1) \log x}{2}\right) d x .
$$

Moreover,

$$
\xi(s)=\xi(1-s) .
$$

Proof. See, for (39), Riemann [2] and, for (40), Landau [18].

2.2. The series representation. The series representation for the entire Riemann zeta-function was first suggested by Edwards [3].

Lemma 2. (Edwards series theorem)

Let $s \in \mathbb{C}$ and $k \in \mathbb{N} \cup\{0\}$. If $\psi(x)$ is defined in (8), then

$$
\xi(s)=\sum_{k=0}^{\infty} \varphi_{k}\left(s-\frac{1}{2}\right)^{2 k}
$$

where

$$
\varphi_{k}=\frac{4}{\Gamma(2 k+1)} \int_{1}^{\infty} \frac{d\left(x^{\frac{3}{2}} \psi^{(1)}(x)\right)}{d x} x^{-\frac{1}{4}}\left(\frac{\log x}{2}\right)^{2 k} d x .
$$

Proof. For the detailed proof, see [3].

2.3. The product representations. We now introduce the products of Hadamard and Patterson and propose the variants of them.

To begin with, we investigate the special values for the entire Riemann zetafunction.

Lemma 3. (Non-vanishing theorem)

There exist

$$
\xi(0)=\xi(1) \neq 0
$$


RIEMANN HYPOTHESIS

10

and

$$
\xi(1 / 2) \neq 0 .
$$

Proof. See [18] and related references ([6], p.288; [46], p.49).

Remark. Here, $\xi(0)=\xi(1)=1 / 2$ and $\xi(1 / 2) \simeq 0.994$ ([46], p.49).

Lemma 4. (Product of Hadamard I)

Let $s \in \mathbb{C}$ and $n \in \mathbb{N}$. Then

$$
\xi(s)=\xi(0) \prod_{n=1}^{\infty}\left(1-\frac{s}{s_{n}}\right),
$$

where the product runs over zeroes $s_{n}$ of (4).

Proof. See Hadamard [8], Edwards ([3], p.47) and Broughan ([46], p.47).

Lemma 5. (Product of Hadamard II)

Let $\gamma$ be the Euler's constant such that

$$
\mu=\log 2+\frac{1}{2} \log \pi-1-\frac{1}{2} \gamma .
$$

Then for $s \in \mathbb{C}$ and $n \in \mathbb{N}$,

$$
\xi(s)=\xi(0) e^{s \mu} \prod_{n=1}^{\infty}\left(1-\frac{s}{s_{n}}\right) e^{s / s_{n}},
$$

where the product runs over zeroes $s_{n}$ of (4).

Proof. See the work of Hadamard [8].

Remark. It is known that (45) and (47) have been proved by Hadamard [8], (45) has been reported in [3], and (47) has been commented in ([6], p.32), discussed by Valiron and Titchmarsh ([48], p.3), and proved by Ingham ([47], p.57-58). For more detailed work, see [46].

Now, we start with the work from Hadamard to Patterson.

Lemma 6. (Products of Patterson)

Let $s \in \mathbb{C}$ and $n \in \mathbb{N}$. Suppose that

$$
\widehat{s_{n}}=R e\left(s_{n}\right)+i \tau_{n},
$$

which are defined in (20). Then

$$
2 \xi(s)=\prod_{n=1}^{\infty}\left(1-\frac{s}{\widehat{s_{n}}}\right)\left(1-\frac{s}{1-\widehat{s_{n}}}\right),
$$

where the product runs over zeroes $\widehat{s_{n}}$. 
Proof. See the work of Patterson ([17], p.34).

We now propose some variants from the products of Hadamard [8] by Lemma 3.

Theorem 1. Let $s \in \mathbb{C}$ and $n \in \mathbb{N}$. Then

$$
\xi(s)=\xi\left(\frac{1}{2}\right) \prod_{n=1}^{\infty}\left(1-\frac{s-\frac{1}{2}}{s_{n}-\frac{1}{2}}\right),
$$

where the product runs over zeroes $s_{n}$ of (4).

Proof. By Lemmas 3 and 4, we show

$$
\begin{aligned}
& \xi(s) \\
& =\xi(0) \prod_{n=1}^{\infty}\left(1-\frac{s}{s_{n}}\right) \\
& =\xi(0) \prod_{n=1}^{\infty}\left(\frac{s_{n}-s}{s_{n}}\right) \\
& =\xi(0) \prod_{n=1}^{\infty}\left(\frac{s_{n}-\frac{1}{2}}{s_{n}-\frac{1}{2}} \cdot \frac{s_{n}-s}{s_{n}}\right) \\
& =\xi(0)\left(\prod_{n=1}^{\infty} \frac{s_{n}-\frac{1}{2}}{s_{n}}\right) \cdot\left(\prod_{n=1}^{\infty} \frac{s_{n}-s}{s_{n}-\frac{1}{2}}\right) \\
& =\xi(0)\left[\prod_{n=1}^{\infty}\left(1-\frac{1}{2 s_{n}}\right)\right] \cdot\left(\prod_{n=1}^{\infty} \frac{s_{n}-s}{s_{n}-\frac{1}{2}}\right) \\
& =\xi(0)\left[\prod_{n=1}^{\infty}\left(1-\frac{1}{2 s_{n}}\right)\right] \cdot\left[\prod_{n=1}^{\infty} \frac{s_{n}-\frac{1}{2}-\left(s-\frac{1}{2}\right)}{s_{n}-\frac{1}{2}}\right] \\
& =\xi\left(\frac{1}{2}\right) \prod_{n=1}^{\infty}\left(1-\frac{s-\frac{1}{2}}{s_{n}-\frac{1}{2}}\right),
\end{aligned}
$$

which is the required result.

Theorem 2. If $\mu$ is defined in (46), then for $s \in \mathbb{C}$ and $n \in \mathbb{N}$,

$$
\xi(s)=\xi\left(\frac{1}{2}\right) e^{s \mu} \prod_{n=1}^{\infty}\left(1-\frac{s-\frac{1}{2}}{s_{n}-\frac{1}{2}}\right) e^{s / s_{n}},
$$

where the product runs over zeroes $s_{n}$ of (4).

Proof. In view of Lemmas 3 and 5, we have

$$
\begin{aligned}
& \xi(s) \\
& =\xi(0) e^{s \mu} \prod_{n=1}^{\infty}\left(1-\frac{s}{s_{n}}\right) e^{s / s_{n}} \\
& =\left(e^{s \mu} \prod_{n=1}^{\infty} e^{s / s_{n}}\right) \cdot\left[\xi(0) \prod_{n=1}^{\infty}\left(1-\frac{s}{s_{n}}\right)\right] .
\end{aligned}
$$


By Lemma 3 and Theorem 1, Eq. (53) can be rewritten in the form

$$
\begin{aligned}
& \xi(s) \\
& =\left(e^{s \mu} \prod_{n=1}^{\infty} e^{s / s_{n}}\right) \cdot\left[\xi(0) \prod_{n=1}^{\infty}\left(1-\frac{s}{s_{n}}\right)\right] \\
& =\left(e^{s \mu} \prod_{n=1}^{\infty} e^{s / s_{n}}\right) \cdot\left[\xi\left(\frac{1}{2}\right) \prod_{n=1}^{\infty}\left(1-\frac{s-\frac{1}{2}}{s_{n}-\frac{1}{2}}\right)\right] \\
& =\xi\left(\frac{1}{2}\right) e^{s \mu} \prod_{n=1}^{\infty}\left(1-\frac{s-\frac{1}{2}}{s_{n}-\frac{1}{2}}\right) e^{s / s_{n}},
\end{aligned}
$$

and the result follows.

We now suggest a variant from the product of Patterson ([17], p.34) by Lemma 3.

Theorem 3. Let $s \in \mathbb{C}$ and $n \in \mathbb{N}$. Then

$$
\xi(s)=\xi\left(\frac{1}{2}\right) \prod_{n=1}^{\infty}\left(1-\frac{\left(s-\frac{1}{2}\right)^{2}}{\left(\widehat{s_{n}}-\frac{1}{2}\right)^{2}}\right)
$$

where the product runs over $\widehat{s_{n}}$, which are defined in (19).

Proof. With (49) we arrive at

$$
\begin{aligned}
& \xi(s) \\
& =\frac{1}{2} \prod_{n=1}^{\infty}\left(1-\frac{s}{\widehat{s_{n}}}\right) \prod_{n=1}^{\infty}\left(1-\frac{s}{1-\widehat{s_{n}}}\right) \\
& =\frac{1}{2}\left[\prod_{n=1}^{\infty}\left(1-\frac{1}{2 \widehat{s_{n}}}\right) \cdot \prod_{n=1}^{\infty}\left(1-\frac{s-\frac{1}{2}}{\widehat{s_{n}}-\frac{1}{2}}\right)\right] \cdot\left[\prod_{n=1}^{\infty}\left(1-\frac{\frac{1}{2}}{1-\widehat{s_{n}}}\right) \cdot \prod_{n=1}^{\infty}\left(1+\frac{s-\frac{1}{2}}{\widehat{s_{n}}-\frac{1}{2}}\right)\right] \\
& =\frac{1}{2}\left[\prod_{n=1}^{\infty}\left(1-\frac{1}{2 \widehat{s_{n}}}\right) \cdot \prod_{n=1}^{\infty}\left(1-\frac{1}{2}\right)\right] \cdot\left[\prod_{n=1}^{\infty}\left(1-\frac{s-\frac{1}{2}}{\widehat{s_{n}}-\frac{1}{2}}\right) \cdot \prod_{n=1}^{\infty}\left(1+\frac{s-\frac{1}{2}}{\widehat{s_{n}}-\frac{1}{2}}\right)\right] \\
& =\frac{1}{2}\left[\prod_{n=1}^{\infty}\left(1-\frac{1}{2 \widehat{s_{n}}}\right) \cdot \prod_{n=1}^{\infty}\left(1-\frac{\frac{1}{2}}{1-\widehat{s_{n}}}\right)\right] \cdot\left[\prod_{n=1}^{\infty}\left(1-\frac{s-\frac{1}{2}}{\widehat{s_{n}}-\frac{1}{2}}\right)\left(1+\frac{s-\frac{1}{2}}{\widehat{s_{n}}-\frac{1}{2}}\right)\right] \\
& =\frac{1}{2}\left[\prod_{n=1}^{\infty}\left(1-\frac{1}{2 \widehat{s_{n}}}\right) \cdot \prod_{n=1}^{\infty}\left(1-\frac{1}{2}\right)\right] \cdot\left[\prod_{n=1}^{\infty}\left(1-\frac{\left(s-\frac{1}{2}\right)^{2}}{\left(\widehat{s_{n}}-\frac{1}{2}\right)^{2}}\right)\right] \\
& =\xi\left(\frac{1}{2}\right) \prod_{n=1}^{\infty}\left(1-\frac{\left(s-\frac{1}{2}\right)^{2}}{\left(\widehat{s_{n}}-\frac{1}{2}\right)^{2}}\right),
\end{aligned}
$$


where

$$
\begin{aligned}
& \prod_{n=1}^{\infty}\left(1-\frac{s}{\widehat{s_{n}}}\right) \\
& =\prod_{n=1}^{\infty}\left(\frac{\widehat{s_{n}}-s}{\widehat{s_{n}}}\right) \\
& =\prod_{n=1}^{\infty}\left(\frac{\widehat{s_{n}}-\frac{1}{2}}{\widehat{s_{n}}-\frac{1}{2}} \cdot \frac{\widehat{s_{n}}-s}{\widehat{s_{n}}}\right) \\
& =\left(\prod_{n=1}^{\infty} \frac{\widehat{s_{n}}-\frac{1}{2}}{\widehat{s_{n}}}\right) \cdot\left(\prod_{n=1}^{\infty} \frac{\widehat{s_{n}}-s}{\widehat{s_{n}}-\frac{1}{2}}\right) \\
& =\left[\prod_{n=1}^{\infty}\left(1-\frac{1}{2 \widehat{s_{n}}}\right)\right] \cdot\left(\prod_{n=1}^{\infty} \frac{\widehat{s_{n}}-s}{\widehat{s}_{n}}-\frac{1}{2}\right) \\
& =\left[\prod_{n=1}^{\infty}\left(1-\frac{1}{2 \widehat{s_{n}}}\right)\right] \cdot\left[\prod_{n=1}^{\infty} \frac{\widehat{s_{n}}-\frac{1}{2}-\left(s-\frac{1}{2}\right)}{\widehat{s_{n}}-\frac{1}{2}}\right] \\
& =\left[\prod_{n=1}^{\infty}\left(1-\frac{1}{2 \widehat{s_{n}}}\right)\right] \cdot\left[\prod_{n=1}^{\infty}\left(1-\frac{s-\frac{1}{2}}{\widehat{s_{n}}-\frac{1}{2}}\right)\right]
\end{aligned}
$$

and

$$
\begin{aligned}
& \prod_{n=1}^{\infty}\left(1-\frac{s}{1-\widehat{s_{n}}}\right) \\
& =\prod_{n=1}^{\infty}\left(\frac{1-\widehat{s_{n}}-s}{1-\widehat{s_{n}}}\right) \\
& =\prod_{n=1}^{\infty}\left(\frac{1-\widehat{s_{n}}-\frac{1}{2}}{1-\widehat{s_{n}}-\frac{1}{2}} \cdot \frac{1-\widehat{s_{n}}-s}{1-\widehat{s_{n}}}\right) \\
& =\left(\prod_{n=1}^{\infty} \frac{1-\widehat{s_{n}}-\frac{1}{2}}{1-\widehat{s_{n}}}\right) \cdot\left(\prod_{n=1}^{\infty} \frac{1-\widehat{s_{n}}-s}{1-\widehat{s_{n}}-\frac{1}{2}}\right) \\
& =\left[\prod_{n=1}^{\infty}\left(1-\frac{\frac{1}{2}}{1-\widehat{s_{n}}}\right)\right] \cdot\left(\prod_{n=1}^{\infty} \frac{1-\widehat{s_{n}}-s}{1-\widehat{s_{n}}-\frac{1}{2}}\right) \\
& =\left[\prod_{n=1}^{\infty}\left(1-\frac{1}{1}-\widehat{s_{n}}\right)\right] \cdot\left[\prod_{n=1}^{\infty} \frac{1-\widehat{s_{n}}-\frac{1}{2}-\left(s-\frac{1}{2}\right)}{1-\widehat{s_{n}}-\frac{1}{2}}\right] \\
& =\left[\prod_{n=1}^{\infty}\left(1-\frac{\frac{1}{2}}{1-\widehat{s_{n}}}\right)\right] \cdot\left[\prod_{n=1}^{\infty}\left(1-\frac{s-\frac{1}{2}}{1-\widehat{s_{n}}-\frac{1}{2}}\right)\right] \\
& =\left[\prod_{n=1}^{\infty}\left(1-\frac{\frac{1}{2}}{1-\widehat{s_{n}}}\right)\right] \cdot\left[\prod_{n=1}^{\infty}\left(1+\frac{s-\frac{1}{2}}{\widehat{s_{n}}-\frac{1}{2}}\right)\right] \text {. }
\end{aligned}
$$

Theorem 4. If $\mu$ is defined in (46), then for $s \in \mathbb{C}$ and $n \in \mathbb{N}$,

$$
e^{s \mu} \prod_{n=1}^{\infty} e^{s / s_{n}}=1,
$$

where the product runs over zeroes $s_{n}$ of (4). 
Proof. By (45), we have

$$
\xi(s)=\xi(0) \prod_{n=1}^{\infty}\left(1-\frac{s}{s_{n}}\right)
$$

and by (47), we show

$$
\xi(s)=\xi(0) e^{s \mu} \prod_{n=1}^{\infty}\left(1-\frac{s}{s_{n}}\right) e^{s / s_{n}} .
$$

Thus, we deduce from (60) and (61) that

$$
\xi(0) \prod_{n=1}^{\infty}\left(1-\frac{s}{s_{n}}\right)=\xi(0) e^{s \mu} \prod_{n=1}^{\infty}\left(1-\frac{s}{s_{n}}\right) e^{s / s_{n}},
$$

which implies that

$$
e^{s \mu} \prod_{n=1}^{\infty} e^{s / s_{n}}=1
$$

So, the proof is completed.

2.4. Order, exponent of convergence, and genus. To show the sufficient condition for the existence of the zeros of the entire Riemann zeta-function, we now introduce the theory of the entire functions.

Let

$$
\mathrm{M}(r)=\max _{r=|s|}|\xi(s)|
$$

be the maximum modulus of $\xi(s)$ on a disk of radius $r$.

We denote the order $\vartheta$ of $\xi(s)$ by

$$
\vartheta=\lim _{r \rightarrow \infty} \sup \frac{\log \log \mathbb{M}(r)}{\log r} .
$$

The genus of the canonical product, $\delta$, is defined by a canonical product

$$
\prod_{n=1}^{\infty} \mathbb{E}\left(s / s_{n}, \delta\right)=\prod_{n=1}^{\infty}\left(1-\frac{s}{s_{n}}\right)=\frac{\xi(s)}{\xi(0)} .
$$

By Lemma 4, we find that $\xi(s)$ is an entire function having infinite number of zeros $s_{n}$. Let

$$
\mathbb{M}=\left\{s_{n}\right\}_{n=1}^{\infty}, \quad\left|s_{n}\right| \leq\left|s_{n+1}\right| \quad(n \in \mathbb{N})
$$


be a sequence of complex numbers with $\lim _{n \rightarrow \infty}\left|s_{n}\right|=+\infty$. The exponent of convergence $\nu$ of the sequence $\mathbb{M}$ is defined by

$$
\nu=\inf \left\{\left.\alpha|| s_{n}\right|^{-\alpha}<\infty, \xi\left(s_{n}\right)=0\right\} .
$$

Theorem 5. $\xi(s)$ is an integral function of order $\vartheta=1$ with the convergence exponent of their zeros $\nu=1$ and genus $\delta=0$.

Proof. Applying Lemmas 3 and 4, we have

$$
\xi(s)=\xi(0) \prod_{n=1}^{\infty}\left(1-\frac{s}{s_{n}}\right)
$$

where $\xi(0) \neq 0$.

Hadamard proved [8] that $\xi(s)$ is an integral function of finite order with genus $\delta=0$. Titchmarsh ([48], p.3) proved that $\xi(s)$ is an integral function of order $\vartheta=1$. By applying the theorem of Borel ([50], p.30; [51], p.19), we find that an entire function of order $\vartheta=1$ is equal to the convergence exponent of its zeros, and we show $\vartheta=\nu=1$.

Thus, the result follows.

Remark. By Theorem 4, we deduce that $\xi(s)$ is of genus $\delta=0$ since $\xi(s)$ is an integral function of order $\vartheta=1$.

2.5. Some equivalent classes. Here, we now present some equivalent classes, which are connected with the series and product representations of them.

Class I

If (40) is valid, by Theorem 1 and Lemma 2, there exist

$$
\sum_{k=0}^{\infty} \varphi_{k}\left(s-\frac{1}{2}\right)^{2 k}=\xi(1 / 2) \prod_{n=1}^{\infty}\left(1-\frac{s-\frac{1}{2}}{s_{n}-\frac{1}{2}}\right),
$$

or, alternatively, by Lemma 2 and Theorem 2 ,

$$
\sum_{k=0}^{\infty} \varphi_{k}\left(s-\frac{1}{2}\right)^{2 k}=\xi\left(\frac{1}{2}\right) \prod_{n=1}^{\infty}\left(1-\frac{\left(s-\frac{1}{2}\right)^{2}}{\left(\widehat{s_{n}}-\frac{1}{2}\right)^{2}}\right)
$$

where $\xi(1 / 2) \neq 0, s \in \mathbb{C}, k \in \mathbb{N} \cup\{0\}, n \in \mathbb{N}, \varphi_{k}$ is defined in (42), $\widehat{s_{n}}$ are defined in (20), and the product runs over zeroes $s_{n}$ of (4).

Class II

If (40) is valid, by Lemmas 2 and 4 , there is

$$
\sum_{k=0}^{\infty} \varphi_{k}\left(s-\frac{1}{2}\right)^{2 k}=\xi(0) \prod_{n=1}^{\infty}\left(1-\frac{s}{s_{n}}\right)
$$


where $\xi(0) \neq 0, s \in \mathbb{C}, k \in \mathbb{N} \cup\{0\}, n \in \mathbb{N}, \varphi_{k}$ is defined in (42), and the product runs over zeroes $s_{n}$ of (4).

Class III

If (40) is valid, by Lemma 4 and Theorems 1, this implies that there exists

$$
\xi(0) \prod_{n=1}^{\infty}\left(1-\frac{s}{s_{n}}\right)=\xi(1 / 2) \prod_{n=1}^{\infty}\left(1-\frac{s-\frac{1}{2}}{s_{n}-\frac{1}{2}}\right),
$$

where $s \in \mathbb{C}, n \in \mathbb{N}, \xi(0) \neq 0(\xi(1 / 2) \neq 0)$, and the products run over zeroes $s_{n}$ of (4).

\section{Theory of the RiEMAnN $\Xi$ FUnCtion}

In this section we study the theory of the Riemann $\Xi$ function. We consider its order, convergence exponent of its zeros, and genus, theorems of Hardy and Ingham, Riemann-von Mangoldt formula and Riemann-Siegel formula. Then, we consider its integral, series and product formulas. Finally, we study its real zeros based on the theorem of Hardy and give the proof of the Riemann hypothesis.

Let us recall the Riemann $\Xi$ function, defined by [2]

$$
\Xi(t)=\xi\left(\frac{1}{2}+i t\right)
$$

3.1. Order, convergence exponent, and genus. Now, we start with its order, convergence exponent, and genus to study the existence of its zeros.

Theorem 6. Let $t \in \mathbb{C}$. Then, $\Xi(t)$ is an integral function of order $\vartheta=1$ with the convergence exponent of their zeros $\nu=1$ and genus $\delta=0$.

Proof. Titchmarsh ([49], p.29) proved that $\Xi(t)$ is an integral function of order $\vartheta=1$.

By (66), we know

$$
\prod_{n=1}^{\infty} \mathbb{E}\left(i t /\left(s_{n}-1 / 2\right), \delta\right)=\prod_{n=1}^{\infty}\left(1-\frac{i t}{s_{n}-\frac{1}{2}}\right)=\frac{\Xi(t)}{\Xi(0)},
$$

and we find $\delta=0$.

By applying the theorem of Borel ([50], p.30; [51], p.19), we have that an entire function of order $\vartheta=1$ is equal to the convergence exponent of its zeros, and we get $\vartheta=\nu=1([49]$, p.30)).

Thus, we finish the proof. 
RIEMANN HYPOTHESIS

3.2. Integral representations. We now report the Riemann, Jensen and Hardy integral representations of it.

Lemma 7. (Riemann integral theorem)

Let $t \in \mathbb{C}$. If $\psi(x)$ is defined in (8), then

$$
\Xi(t)=4 \int_{1}^{\infty} \frac{d\left(x^{\frac{3}{2}} \psi^{(1)}(x)\right)}{d x} x^{-\frac{1}{4}} \cos \left(\frac{t \log x}{2}\right) d x
$$

or, alternatively,

$$
\Xi(t)=\frac{1}{2}-\left(t^{2}+\frac{1}{4}\right) \int_{1}^{\infty} \psi(x) x^{-\frac{3}{4}} \cos \left(\frac{t}{2} \log x\right) d x .
$$

Moreover,

$$
\Xi(-t)=\Xi(t)
$$

Proof. See [2].

Lemma 8. (Jensen integral theorem)

Let $t \in \mathbb{R}$. Then,

$$
\Xi(t)=\int_{0}^{\infty} \mathbb{k}(x) \cos (t x) d x
$$

where

$$
\mathbb{k}(x)=4 \sum_{n=1}^{\infty}\left(2 n^{4} \pi^{2} e^{9 x / 2}-3 n^{2} \pi e^{5 x / 2}\right) e^{-n^{2} \pi e^{2 x}} .
$$

Proof. See the work of Jensen $[52,53]$.

Remark. Wintner [54] proved that

$$
\mathbb{k}(x)>0
$$

and

$$
\mathbb{k}(x)=\mathbb{k}(-x) .
$$

Lemma 9. (Hardy integral theorem)

Let $t \in \mathbb{R}$. Then,

$$
\mathbb{S}(x)=\int_{0}^{\infty} \frac{\Xi(t)}{t^{2}+\frac{1}{4}} \cos (t x) d x
$$


where

$$
\mathbb{S}(x)=\frac{\pi}{2}\left(e^{x / 2}-2 e^{-x / 2} \psi\left(e^{-2 x}\right)\right),
$$

and $\psi(x)$ is defined in (8). Moreover,

$$
\mathbb{S}(-x)=\mathbb{S}(x) .
$$

Proof. See [9, 55] and the Titchmarsh's book ([49], p.260).

Remark. The Riemann integral theorem [2] was used to obtain the existence of the real zeros [3]. The Jensen integral theorem [52, 53] was presented to get the Pólya series theorem [13], which is related to the Jensen polynomials for the Riemann zetafunction [45]. The Hardy integral theorem [9] was considered to obtain the theorem of Hardy $[9,55]$.

3.3. Series representations. We now investigate the series representations for the Riemann $\Xi$ function.

Lemma 10. (Hadamard series theorem)

Let $t \in \mathbb{R}$. Then,

$$
\Xi(t)=\sum_{k=0}^{\infty}(-1)^{k} \varphi_{k} t^{2 k}
$$

and

$$
\varphi_{k}=\frac{D_{2 k}}{4}-D_{2 k-2}
$$

where

$$
D_{k}=\frac{1}{2^{2 k} \Gamma(2 k+1)} \int_{1}^{\infty} \psi(x) x^{-\frac{3}{4}}(\log x)^{k} d x .
$$

Proof. See [8].

Lemma 11. (Pólya series theorem)

Let $t \in \mathbb{R}$. Then,

$$
\Xi(t)=\sum_{k=0}^{\infty}(-1)^{k} \varphi_{k} t^{2 k}
$$

and

$$
\varphi_{k}=\frac{2}{\pi \Gamma(2 k+1)} \int_{0}^{\infty} \mathbb{k}(x) x^{2 k} d x=\frac{\Xi^{(2 m)}(0)}{\Gamma(2 k+1)} .
$$


RIEMANN HYPOTHESIS

19

Proof. See [13].

Theorem 7. Let $t \in \mathbb{R}$. Then,

$$
\Xi(t)=\sum_{k=0}^{\infty}(-1)^{k} \varphi_{k} t^{2 k}
$$

and

$$
\varphi_{k}=\frac{4}{\Gamma(2 k+1)} \int_{1}^{\infty} \frac{d\left(x^{\frac{3}{2}} \psi^{(1)}(x)\right)}{d x} x^{-\frac{1}{4}}\left(\frac{\log x}{2}\right)^{2 k} d x
$$

Proof. Substituting $s=1 / 2+i t$ into Lemma 2, the result follows.

Remark. The coefficients of the series read

$$
\begin{aligned}
& \varphi_{k}=\frac{D_{2 k}}{4}-D_{2 k-2} \\
& =\frac{1}{2^{2 k} \Gamma(2 k+1)} \int_{1}^{\infty} \psi(x) x^{-\frac{3}{4}}(\log x)^{k} d x \\
& =\frac{2}{\pi \Gamma(2 k+1)} \int_{0}^{\infty} \mathrm{k}(x) x^{2 k} d x \\
& =\frac{\Xi^{(2 k)}(0)}{\Gamma(2 k+1)} \\
& =\frac{4}{\Gamma(2 k+1)} \int_{1}^{\infty} \frac{d\left(x^{\frac{3}{2}} \psi^{(1)}(x)\right)}{d x} x^{-\frac{1}{4}}\left(\frac{\log x}{2}\right)^{2 k} d x
\end{aligned}
$$

with $\varphi_{k}>0$, where (88) is valid.

By Theorem 6, the coefficients of the series, obtained by Hadamard series theorem, Pólya series theorem and Theorem $\%$, is related to order $\vartheta$ of the entire function $\Xi(t)$ by ([51], Theorem 2.2.2, p.9)

$$
\vartheta=\lim _{k \rightarrow \infty} \sup \frac{\log \log k}{\log \left(1 /\left|\varphi_{k}\right|\right)}=1,
$$

and the convergence exponent of its zeros $\nu$ by

$$
\nu=\lim _{k \rightarrow \infty} \sup \frac{\log \log k}{\log \left(1 /\left|\varphi_{k}\right|\right)}=1
$$

if one uses the theorem of Borel ([50], p.30; [51], p.19). Moreover, it is related to the formula of Jensen, which is connected with the number of zeros of $\Xi(t)$ ([23], p.14). 
RIEMANN HYPOTHESIS

3.4. Product formulas. We now consider the products for the Riemann $\Xi$ function.

Lemma 12. Let $t \in \mathbb{R}$ and $n \in \mathbb{N}$. Then

$$
\Xi(t)=\Xi(0) \prod_{n=1}^{\infty}\left(1-\frac{t^{2}}{\tau_{n}^{2}}\right)
$$

where $\tau_{n}$ run the positive roots of $\Xi(t)=0$. Moreover,

$$
\log \Xi(t)=\log \Xi(0)+\log \sum_{n=1}^{\infty}\left(1-\frac{t^{2}}{\tau_{n}^{2}}\right) .
$$

Proof. (94) was reported by Cahen [15] and Titchmarsh [16]. (95) was proposed by Riemann [2].

Lemma 13. Let $t \in \mathbb{R}$ and $n \in \mathbb{N}$. Then

$$
\Xi(t)=\Xi(0) \prod_{n=1}^{\infty}\left(1-\frac{t}{t_{n}}\right)
$$

where $t_{n}$ run the roots of $\Xi(t)=0$.

Proof. (96) was proposed by Hadamard [8] and discussed by Landau ([6], p.199).

Theorem 8. Let $t \in \mathbb{R}$ and $n \in \mathbb{N}$. Then

$$
\Xi(t)=\Xi(0) \prod_{n=1}^{\infty}\left(1-\frac{i t}{s_{n}-\frac{1}{2}}\right),
$$

where $s_{n}$ run the roots of $\xi(s)=0$.

Proof. Taking $s=1 / 2+i t$ into Lemma 4 , we present

$$
\Xi(t)=\xi(1 / 2+i t)=\xi(0) \prod_{n=1}^{\infty}\left(1-\frac{\frac{1}{2}+i t}{s_{n}}\right)
$$


Hence,

$$
\begin{aligned}
& \Xi(t)=\xi(0) \prod_{n=1}^{\infty}\left(1-\frac{\frac{1}{2}+i t}{s_{n}}\right) \\
& =\xi(0) \prod_{n=1}^{\infty}\left(\frac{s_{n}-\frac{1}{2}-i t}{s_{n}}\right) \\
& =\xi(0) \prod_{n=1}^{\infty}\left(\frac{s_{n}-\frac{1}{2}}{s_{n}-\frac{1}{2}} \cdot \frac{s_{n}-\frac{1}{2}-i t}{s_{n}}\right) \\
& =\xi(0)\left(\prod_{n=1}^{\infty} \frac{s_{n}-\frac{1}{2}}{s_{n}}\right) \cdot\left(\prod_{n=1}^{\infty} \frac{s_{n}-\frac{1}{2}-i t}{s_{n}-\frac{1}{2}}\right) \\
& =\xi(0)\left[\prod_{n=1}^{\infty}\left(1-\frac{1}{2 s_{n}}\right)\right] \cdot\left[\prod_{n=1}^{\infty} \frac{s_{n}-\frac{1}{2}-i t}{s_{n}-\frac{1}{2}}\right] \\
& =\xi\left(\frac{1}{2}\right) \prod_{n=1}^{\infty}\left(1-\frac{i t}{s_{n}-\frac{1}{2}}\right) \\
& =\Xi(0) \prod_{n=1}^{\infty}\left(1-\frac{i t}{s_{n}-\frac{1}{2}}\right),
\end{aligned}
$$

and the required result follows.

Theorem 9. If $\mu$ is defined in (46), then for $t \in \mathbb{R}$ and $n \in \mathbb{N}$,

$$
\Xi(s)=\Xi(0) e^{(1 / 2+i t) \mu} \prod_{n=1}^{\infty}\left(1-\frac{i t}{s_{n}-\frac{1}{2}}\right) e^{(1 / 2+i t) / s_{n}},
$$

where the product runs over zeroes $s_{n}$ of (4).

Proof. Taking $s=1 / 2+i t$ into Lemma 5 , we give

$$
\Xi(t)=\xi(1 / 2+i t)=\xi(0) e^{\mu(1 / 2+i t)} \prod_{n=1}^{\infty}\left(1-\frac{\frac{1}{2}+i t}{s_{n}}\right) e^{(1 / 2+i t) / s_{n}} .
$$

Hence, by Theorem 8, we show

$$
\begin{aligned}
& \Xi(t) \\
& =\xi(0) e^{\mu(1 / 2+i t)} \prod_{n=1}^{\infty}\left(1-\frac{\frac{1}{2}+i t}{s_{n}}\right) e^{(1 / 2+i t) / s_{n}} \\
& =\left(e^{\mu(1 / 2+i t)} \prod_{n=1}^{\infty} e^{(1 / 2+i t) / s_{n}}\right) \cdot\left[\xi(0) \prod_{n=1}^{\infty}\left(1-\frac{\frac{1}{2}+i t}{s_{n}}\right)\right] \\
& =\left(e^{\mu(1 / 2+i t)} \prod_{n=1}^{\infty} e^{(1 / 2+i t) / s_{n}}\right) \cdot\left[\Xi(0) \prod_{n=1}^{\infty}\left(1-\frac{i t}{s_{n}-\frac{1}{2}}\right)\right] \\
& =\Xi(0) e^{(1 / 2+i t) \mu} \prod_{n=1}^{\infty}\left(1-\frac{i t}{s_{n}-\frac{1}{2}}\right) e^{(1 / 2+i t) / s_{n}} .
\end{aligned}
$$

Thus, we finish the proof. 
Theorem 10. Let $t \in \mathbb{R}$ and $n \in \mathbb{N}$. Then

$$
\Xi(t)=\Xi(0) \prod_{n=1}^{\infty}\left[1+\frac{t^{2}}{\left(\widehat{s_{n}}-\frac{1}{2}\right)^{2}}\right],
$$

where the product runs over $\widehat{s_{n}}$, which are defined in (20).

Proof. Taking $s=1 / 2+i t$ into (49), we arrive at

$$
\Xi(t)=\xi(1 / 2+i t)=\frac{1}{2} \prod_{n=1}^{\infty}\left(1-\frac{\frac{1}{2}+i t}{\widehat{s_{n}}}\right) \prod_{n=1}^{\infty}\left(1-\frac{\frac{1}{2}+i t}{1-\widehat{s_{n}}}\right) .
$$

Therefore,

$$
\begin{aligned}
& \xi(s) \\
& =\frac{1}{2} \prod_{n=1}^{\infty}\left(1-\frac{\frac{1}{2}+i t}{\widehat{s_{n}}}\right) \prod_{n=1}^{\infty}\left(1-\frac{\frac{1}{2}+i t}{1-\widehat{s_{n}}}\right) \\
& =\frac{1}{2}\left[\prod_{n=1}^{\infty}\left(1-\frac{1}{2 \widehat{s_{n}}}\right) \cdot \prod_{n=1}^{\infty}\left(1-\frac{i t}{\widehat{s_{n}}-\frac{1}{2}}\right)\right] \cdot\left[\prod_{n=1}^{\infty}\left(1-\frac{\frac{1}{2}}{1-\widehat{s_{n}}}\right) \cdot \prod_{n=1}^{\infty}\left(1+\frac{i t}{\widehat{s_{n}}-\frac{1}{2}}\right)\right] \\
& =\frac{1}{2}\left[\prod_{n=1}^{\infty}\left(1-\frac{1}{2 \widehat{s_{n}}}\right) \cdot \prod_{n=1}^{\infty}\left(1-\frac{\frac{1}{2}}{1-\widehat{s_{n}}}\right)\right] \cdot\left[\prod_{n=1}^{\infty}\left(1-\frac{i t}{\widehat{s_{n}}-\frac{1}{2}}\right) \cdot \prod_{n=1}^{\infty}\left(1+\frac{i t}{\widehat{s_{n}}-\frac{1}{2}}\right)\right] \\
& =\frac{1}{2}\left[\prod_{n=1}^{\infty}\left(1-\frac{1}{2 \widehat{s_{n}}}\right) \cdot \prod_{n=1}^{\infty}\left(1-\frac{\frac{1}{2}}{1-\widehat{s_{n}}}\right)\right] \cdot\left[\prod_{n=1}^{\infty}\left(1-\frac{i t}{\widehat{s_{n}}-\frac{1}{2}}\right)\left(1+\frac{i t}{\widehat{s_{n}}-\frac{1}{2}}\right)\right] \\
& =\frac{1}{2}\left[\prod_{n=1}^{\infty}\left(1-\frac{1}{2 \widehat{s_{n}}}\right) \cdot \prod_{n=1}^{\infty}\left(1-\frac{\frac{1}{2}}{1-\widehat{s_{n}}}\right)\right] \cdot\left[\prod_{n=1}^{\infty}\left(1+\frac{t^{2}}{\left(\widehat{s_{n}}-\frac{1}{2}\right)^{2}}\right)\right] \\
& =\xi\left(\frac{1}{2}\right) \prod_{n=1}^{\infty}\left(1+\frac{t^{2}}{\left(\widehat{s_{n}}-\frac{1}{2}\right)^{2}}\right) \\
& =\Xi(0) \prod_{n=1}^{\infty}\left[1+\frac{t^{2}}{\left(\widehat{s_{n}}-\frac{1}{2}\right)^{2}}\right] \text {, }
\end{aligned}
$$

where

$$
\begin{aligned}
& \prod_{n=1}^{\infty}\left(1-\frac{\frac{1}{2}+i t}{\widehat{s_{n}}}\right) \\
& =\prod_{n=1}^{\infty}\left(\frac{\widehat{s_{n}}-\frac{1}{2}-i t}{\widehat{\widehat{s}_{n}}}\right) \\
& =\prod_{n=1}^{\infty}\left(\frac{\widehat{s_{n}}-\frac{1}{2}}{\widehat{s_{n}}-\frac{1}{2}} \cdot \frac{\widehat{s_{n}}-\frac{1}{2}-i t}{\widehat{s_{n}}}\right) \\
& =\left(\prod_{n=1}^{\infty} \frac{\widehat{s_{n}}-\frac{1}{2}}{\widehat{s_{n}}}\right) \cdot\left(\prod_{n=1}^{\infty} \frac{\widehat{s_{n}}-\frac{1}{2}-i t}{\widehat{s_{n}}-\frac{1}{2}}\right) \\
& =\left[\prod_{n=1}^{\infty}\left(1-\frac{1}{2 \widehat{s_{n}}}\right)\right] \cdot\left(\prod_{n=1}^{\infty} \frac{\widehat{s_{n}}-\frac{1}{2}-i t}{\widehat{s_{n}}-\frac{1}{2}}\right) \\
& =\left[\prod_{n=1}^{\infty}\left(1-\frac{1}{2 \widehat{s_{n}}}\right)\right] \cdot\left[\prod_{n=1}^{\infty}\left(1-\frac{i t}{\widehat{s_{n}}-\frac{1}{2}}\right)\right]
\end{aligned}
$$


and

$$
\begin{aligned}
& \prod_{n=1}^{\infty}\left(1-\frac{\frac{1}{2}+i t}{1-\widehat{s_{n}}}\right) \\
& =\prod_{n=1}^{\infty}\left(\frac{1-\widehat{s_{n}}-\frac{1}{2}-i t}{1-\widehat{s_{n}}}\right) \\
& =\prod_{n=1}^{\infty}\left(\frac{1-\widehat{s_{n}}-\frac{1}{2}}{1-\widehat{s_{n}}-\frac{1}{2}} \cdot \frac{1-\widehat{s_{n}}-\frac{1}{2}-i t}{1-\widehat{s_{n}}}\right) \\
& =\left(\prod_{n=1}^{\infty} \frac{1-\widehat{s_{n}}-\frac{1}{2}}{1-\widehat{s_{n}}}\right) \cdot\left(\prod_{n=1}^{\infty} \frac{1-\widehat{s_{n}}-\frac{1}{2}-i t}{1-\widehat{s_{n}}-\frac{1}{2}}\right) \\
& =\left[\prod_{n=1}^{\infty}\left(1-\frac{1}{1-\widehat{s_{n}}}\right)\right] \cdot\left[\prod_{n=1}^{\infty} \frac{1-\widehat{s_{n}}-\frac{1}{2}-i t}{1-\widehat{s_{n}}-\frac{1}{2}}\right] \\
& =\left[\prod_{n=1}^{\infty}\left(1-\frac{\frac{1}{2}}{1-\widehat{s_{n}}}\right)\right] \cdot\left[\prod_{n=1}^{\infty}\left(1-\frac{i t}{1-\widehat{s_{n}}-\frac{1}{2}}\right)\right] \\
& =\left[\prod_{n=1}^{\infty}\left(1-\frac{\frac{1}{2}}{1-\widehat{s_{n}}}\right)\right] \cdot\left[\prod_{n=1}^{\infty}\left(1+\frac{i t}{\widehat{s_{n}}-\frac{1}{2}}\right)\right] .
\end{aligned}
$$

Thus, the proof is completed.

3.5. Well-known results. By Theorem 6 , it is seen that $\Xi(t)$ has infinity many zeros. We need to know $\Xi(t)$ has an infinity of real zeros. We now investigate the Riemann-von Mangoldt formula and Riemann-Siegel formula and theorems of Hardy and Ingham.

Lemma 14. (Theorem of Hardy)

There are infinitely many zeros of $\zeta(s)$ on the critical line $\operatorname{Re}(s)=1 / 2$.

Proof. See [9] and ([49], p.254-282).

Lemma 15. (Theorem of Ingham)

There are infinitely many zeros of $\xi(s)$ on the critical line $\operatorname{Re}(s)=1 / 2$.

Proof. See the proof ([47], p.48).

Lemma 16. (Riemann-von Mangoldt formula)

Let

$$
N_{0}(T)=\#\{s=1 / 2+i t: 0 \leq t \leq T, \xi(s)=0\}
$$

Then,

$$
N_{0}(T)=\frac{T}{2 \pi} \log \frac{T}{2 \pi e}+O(\log T) .
$$

Proof. See the work of von Mangoldt [26]. 
Lemma 17. (Riemann-Siegel formula)

Let

$$
e^{i \mathbb{A}(t)} \zeta\left(\frac{1}{2}+i t\right)=2 \sum_{j=1}^{g(t)} \frac{1}{\sqrt{j}} \cos (\mathbb{A}(t)-t \log j)+\mathbb{S}(t)
$$

where $t>0$,

$$
\begin{gathered}
g(t)=\left[\sqrt{\frac{t}{2 \pi}}\right] \\
\mathbb{A}(t)=-\frac{t}{2} \log \pi+\arg \Gamma\left(\frac{1}{4}+\frac{i t}{2}\right)
\end{gathered}
$$

and

Then, the Riemann-Siegel zeros $\tau_{n}$ for $n \in \mathbb{N}$ are determined by (110).

Proof. See the work of Siegel [27].

3.6. All zeros are real. Lemmas 12 and 13 showed $\Xi(t)$ has an infinity of real zeros, which was not proved by Riemann [2], Cahen [15], and Titchmarsh [16]. This problem was reported by Titchmarsh ([48], p.44; [49], p.255). Theorem 6 gives us to show that $\Xi(t)$ has an infinity of zeros. The theorems of Hardy and Ingham imply that infinitely many zeros lie on the critical line $R e(s)=1 / 2$ but do not confirm all zeros lie on the critical line $\operatorname{Re}(s)=1 / 2$. Riemann and Siegel [27] discovered the Riemann-Siegel zeros $t_{n}$. Riemann and von Mangoldt [26] presented that the number of the zeros of $\Xi(t)$. The Jensen polynomials for the Riemann zeta-function have real zeros [45] but the case of all zeros of $\Xi(t)$ has not been known. However, this statement of the conjecture of Jensen [7, 35, 45], that all the zeros of $\Xi(t)$ are real, remains unproved to this day. Now, our aim is to prove it.

Theorem 11. (Conjecture of Jensen)

Let $t \in \mathbb{R}$. Then, all zeros of $\Xi(t)$ are real.

Proof. Now, by Theorem 8, we structure

$$
\Xi(t)=\Xi(0) \prod_{n=1}^{\infty}\left(1-\frac{i t}{s_{n}-\frac{1}{2}}\right)
$$

where $t \in \mathbb{R}$ and $n \in \mathbb{N}$. 
To prove it, our aim is to consider two cases are that all zeros are complex number $\widehat{s_{n}}-\frac{1}{2}=\sigma_{n}+i g_{n}$ for $\sigma_{n} \in \mathbb{R} \backslash\{0\}$ and $g_{n} \in \mathbb{R} \backslash\{0\}$, and that $\widehat{s_{n}}-\frac{1}{2}=f_{n}$ for $f_{n} \in \mathbb{R} \backslash\{0\}$. If they are false, all zeros of $\Xi(t)$ are real.

Now, we begin with the followings:

Case I. We consider the hypothesis that $\Xi(t)$ has the complex zeros, given by

$$
\widehat{s_{n}}-\frac{1}{2}=\sigma_{n}+i g_{n}
$$

where $\sigma_{n} \in \mathbb{R} \backslash\{0\}$ and $g_{n} \in \mathbb{R} \backslash\{0\}$.

Putting (115) into (114), we get

$$
\Xi(t)=\Xi(0) \prod_{n=1}^{\infty}\left(1-\frac{i t}{\sigma_{n}+i g_{n}}\right) .
$$

Substituting $t=(s-1 / 2) / i$ into (116), we have

$$
\xi(s)=\Xi((s-1 / 2) / i)=\Xi(0) \prod_{n=1}^{\infty}\left(1-\frac{s-1 / 2}{\sigma_{n}+i g_{n}}\right)=\xi\left(\frac{1}{2}\right) \prod_{n=1}^{\infty}\left(1-\frac{s-1 / 2}{\sigma_{n}+i g_{n}}\right) .
$$

Since $s_{n}=\operatorname{Re}\left(s_{n}\right)+i t_{n}$ with $\xi\left(s_{n}\right)=0$, where $\operatorname{Re}\left(s_{n}\right) \in \mathbb{R} \backslash\{0\}$ and $t_{n} \in \mathbb{R} \backslash\{0\}$, (117) can be rewritten as

$$
\xi\left(\frac{1}{2}\right) \prod_{n=1}^{\infty}\left(1-\frac{s_{n}-1 / 2}{\sigma_{n}+i g_{n}}\right)=0
$$

which leads to

$$
\sigma_{n}+i g_{n}-\left(s_{n}-1 / 2\right)=0
$$

For $\sigma_{n} \in \mathbb{R} \backslash\{0\}$ and $g_{n} \in \mathbb{R} \backslash\{0\}$ we get

$$
s_{n}=\operatorname{Re}\left(s_{n}\right)+i t_{n}=\sigma_{n}+1 / 2+i g_{n} .
$$

By $\sigma_{n} \in \mathbb{R} \backslash\{0\}$ and (120), we show that the critical line is $\operatorname{Re}\left(s_{n}\right)=\sigma_{n}+1 / 2$, which is in contradiction with the fact that the theorems of Hardy and Ingham imply that infinitely many zeros lie on the critical line $\operatorname{Re}(s)=1 / 2$.

Case II. Now, we give the hypothesis that $\Xi(t)$ has the zeros

$$
\widehat{s_{n}}-\frac{1}{2}=f_{n},
$$

where $f_{n} \in \mathbb{R} \backslash\{0\}$.

In a similar way, substituting (121) into (114), we suggest that

$$
\Xi(t)=\Xi(0) \prod_{n=1}^{\infty}\left(1-\frac{i t}{f_{n}}\right) .
$$


Combining $t=(s-1 / 2) / i$ and (122), we arrive at

$$
\xi(s)=\Xi((s-1 / 2) / i)=\Xi(0) \prod_{n=1}^{\infty}\left(1-\frac{s-1 / 2}{f_{n}}\right)=\xi\left(\frac{1}{2}\right) \prod_{n=1}^{\infty}\left(1-\frac{s-1 / 2}{f_{n}}\right) .
$$

Let $s_{n}=\operatorname{Re}\left(s_{n}\right)+i t_{n}$ with $\operatorname{Re}\left(s_{n}\right) \in \mathbb{R} \backslash\{0\}$ and $t_{n} \in \mathbb{R} \backslash\{0\}$ such that $\xi\left(s_{n}\right)=0$.

From (123) we have

$$
\xi\left(\frac{1}{2}\right) \prod_{n=1}^{\infty}\left(1-\frac{s_{n}-1 / 2}{f_{n}}\right)=0
$$

which yields that

$$
f_{n}-\left(s_{n}-1 / 2\right)=0 .
$$

Since $f_{n} \in \mathbb{R} \backslash\{0\}$, by (125), we show

$$
s_{n}=f_{n}+1 / 2 \in \mathbb{R},
$$

which is in contradiction with the fact $s_{n}=R e\left(s_{n}\right)+i t_{n}$ with $R e\left(s_{n}\right) \in \mathbb{R} \backslash\{0\}$ and $t_{n} \in \mathbb{R} \backslash\{0\}$ since (126) do not belong to the nontrivial zeros of $\zeta(s)$. Moreover, (126) are also in contradiction with the theorems of Hardy and Ingham.

Because two cases are false, all zeros of $\Xi(t)$ are real.

Thus, the result follows.

3.7. A detailed proof of Conjecture 1. Here, our target is to prove that $\operatorname{Re}\left(\widehat{s_{n}}\right)=$ $1 / 2$ and $\operatorname{Re}\left(s_{n}\right)=1 / 2$ since all zeros of $\Xi(t)$ are real.

By Theorem 9, we structure for $t \in \mathbb{R}$ and $n \in \mathbb{N}$,

$$
\Xi(t)=\Xi(0) e^{(1 / 2+i t) \mu} \prod_{n=1}^{\infty}\left(1-\frac{i t}{s_{n}-\frac{1}{2}}\right) e^{(1 / 2+i t) / s_{n}},
$$

where $\mu$ is defined in (46) and the product runs over zeroes $s_{n}$ of (4).

Combining (127) and Theorem 11, we find that there exist real zeros $\widetilde{\tau_{n}} \in \mathbb{R}$ of $\Xi(t)$ such that

$$
\Xi\left(\widetilde{\tau_{n}}\right)=\Xi(0) e^{\left(1 / 2+i \widetilde{\tau_{n}}\right) \mu} \prod_{n=1}^{\infty}\left(1-\frac{i \widetilde{\tau_{n}}}{s_{n}-\frac{1}{2}}\right) e^{\left(1 / 2+i \widetilde{\tau_{n}}\right) / s_{n}}=0,
$$

where $\mu$ is defined in (46) and the product runs over zeroes $s_{n}$ of (4).

Now, by (128),

$$
1-\frac{i \widetilde{\tau_{n}}}{s_{n}-\frac{1}{2}}=0
$$


RIEMANN HYPOTHESIS

which implies that

$$
s_{n}=\frac{1}{2}+i \widetilde{\tau_{n}}
$$

With (130), we have

$$
R e\left(s_{n}\right)=\frac{1}{2},
$$

and

$$
\operatorname{Im}\left(s_{n}\right)=t_{n}=\widetilde{\tau_{n}}
$$

Thus,

$$
\tau_{n}=\left|t_{n}\right|=\left|\widetilde{\tau_{n}}\right|
$$

and

$$
s_{n}=\frac{1}{2}+i t_{n}=\frac{1}{2}+i \widetilde{\tau_{n}}=\frac{1}{2} \pm \tau_{n} .
$$

By Lemma 17, the Riemann-Siegel formula gives all values of (132) and the number of all real zeros is confirmed by the Riemann-von Mangoldt formula.

Thus, we finish the proof of Conjecture 1.

Remark. By Theorem 8, we also obtain the same result.

3.8. Turán inequalities. The Turán inequalities was proved by many researchers $[11,12,13]$. Here, we give a new proof of the Turán inequalities from the point of view of theory of the entire functions.

Theorem 12. (Turán inequalities)

Let $k \in \mathbb{N} \cup\{0\}$. Then,

$$
\varphi_{k}^{2}-\left(\frac{2 k-1}{2 k+1}\right) \varphi_{k-1} \varphi_{k+1}>0
$$

Proof. By Theorem 6, $\Xi(t)$ is an even integral function of order $\vartheta=1$ and real for real $t$. With Theorem 11, $\Xi(t)$ has only real zeros. From Lemma 10, Theorem 7 and Lemma 11, we show

$$
\Xi(t)=\sum_{k=0}^{\infty}(-1)^{k} \varphi_{k} t^{2 k} .
$$

By theory of the entire functions ([51], Theorem 2.8.2., p.24), we arrive at

$$
\varphi_{k}^{2}-\left(\frac{2 k-1}{2 k+1}\right) \varphi_{k-1} \varphi_{k+1}>0
$$

Thus, the proof is completed. 
3.9. Proofs of Lemmas 12 and 13. There is the exact significance of this remark, reported by Titchmarsh ([48], p.44; [49], p.255), that Riemann [2] considered that $\tau_{n}$ are real. Riemann [2] and Cahen [15] did not prove it. Hadamard [8] and Landau ([6], p.199) did not report the proof of it. Here, we give their proofs.

At first, we prove Lemma 12.

Putting (133) into Theorem 10, we show

$$
\begin{aligned}
& \Xi(t)=\Xi(0) \prod_{n=1}^{\infty}\left[1+\frac{t^{2}}{\left(\widehat{s_{n}}-\frac{1}{2}\right)^{2}}\right] \\
& =\Xi(0) \prod_{n=1}^{\infty}\left\{1+\frac{t^{2}}{\left[\left(\frac{1}{2}+i \tau_{n}\right)-\frac{1}{2}\right]^{2}}\right\} \\
& =\Xi(0) \prod_{n=1}^{\infty}\left(1-\frac{t^{2}}{\tau_{n}^{2}}\right)
\end{aligned}
$$

and

$$
\begin{aligned}
& \log \Xi(t)=\log \Xi(0)+\log \sum_{n=1}^{\infty}\left[1+\frac{t^{2}}{\left(\widehat{s_{n}}-\frac{1}{2}\right)^{2}}\right] \\
& =\log \Xi(0)+\log \sum_{n=1}^{\infty}\left\{1+\frac{t^{2}}{\left[\left(\frac{1}{2}+i \tau_{n}\right)-\frac{1}{2}\right]^{2}}\right\}^{\infty} \\
& =\log \Xi(0)+\log \sum_{n=1}^{\infty}\left(1-\frac{t^{2}}{\tau_{n}^{2}}\right),
\end{aligned}
$$

which are the required result.

Now, we give the proof of Lemma 13.

Combining (134) and Theorem 8, we give

$$
\begin{aligned}
& \Xi(t) \\
& =\Xi(0) \prod_{n=1}^{\infty}\left(1-\frac{i t}{s_{n}-\frac{1}{2}}\right) \\
& =\Xi(0) \prod_{n=1}^{\infty}\left[1-\frac{i t}{\left(\frac{1}{2}+i t_{n}\right)-\frac{1}{2}}\right] \\
& =\Xi(0) \prod_{n=1}^{\infty}\left(1-\frac{i t}{i t_{n}}\right) \\
& =\Xi(0) \prod_{n=1}^{\infty}\left(1-\frac{t}{t_{n}}\right)
\end{aligned}
$$

and the required result follows.

3.10. A equivalent class. We now discuss a equivalent class, which is connected with the series and product representations.

By Lemmas 10 and 13, there exist 


$$
\sum_{k=0}^{\infty}(-1)^{k} \varphi_{k} t^{2 k}=\Xi(0) \prod_{n=1}^{\infty}\left(1-\frac{t}{t_{n}}\right),
$$

or, alternatively, by Lemmas 10 and 12,

$$
\sum_{k=0}^{\infty}(-1)^{k} \varphi_{k} t^{2 k}=\Xi(0) \prod_{n=1}^{\infty}\left(1-\frac{t^{2}}{\tau_{n}^{2}}\right)
$$

where $\Xi(0) \neq 0, s \in \mathbb{C}, k \in \mathbb{N} \cup\{0\}, n \in \mathbb{N}, \varphi_{k}$ is defined in (93), $t_{n}$ run the roots of $\Xi(t)=0$ and $\tau_{n}$ run the positive roots of $\Xi(t)=0$.

\section{Comments}

4.1. Nontrivial zeros. By applying Lemma 12 and Theorem 10, we also obtain

$$
\Xi(t)=\Xi(0) \prod_{n=1}^{\infty}\left[1+\frac{t^{2}}{\left(\widehat{s_{n}}-\frac{1}{2}\right)^{2}}\right]=\Xi(0) \prod_{n=1}^{\infty}\left(1-\frac{t^{2}}{\tau_{n}^{2}}\right),
$$

which yields that

$$
\widehat{s_{n}}=\frac{1}{2} \pm \tau_{n}
$$

It is observed that $s_{n}=1 / 2+i t_{n}, 1-s_{n}=1 / 2-i t_{n}, \overline{s_{n}}=1 / 2-i t_{n}$ and $1-\overline{s_{n}}=$ $1 / 2+i t_{n}$ are the zeros (nontrivial zeros) for the entire Riemann zeta-function, where $t_{n}= \pm \tau_{n}$ since all zeros are nontrivial zeros.

4.2. Some equivalence theorems. We now present some equivalence theorems associated with the Riemann zeta-function.

Theorem 13. (Equivalence theorem I)

Let $s \in \mathbb{C}$ and $n \in \mathbb{N}$. Then the following representations are equivalent:

A1:

$$
\xi(s)=\xi(0) \prod_{n=1}^{\infty}\left(1-\frac{s}{s_{n}}\right),
$$

where the product runs over zeroes $s_{n}$ of (4);

\section{A2:}

$$
\xi(s)=\xi(1 / 2) \prod_{n=1}^{\infty}\left(1+\frac{i(s-1 / 2)}{t_{n}}\right),
$$

where $t_{n}$ run the roots of $\Xi(t)=0$; 
A3:

$$
\xi(s)=\xi(0) e^{s \mu} \prod_{n=1}^{\infty}\left(1-\frac{s}{s_{n}}\right) e^{s / s_{n}}
$$

where $\mu$ is defined in (46) and the product runs over zeroes $s_{n}$ of (4);

A4:

$$
\xi(s)=\xi\left(\frac{1}{2}\right) e^{s \mu} \prod_{n=1}^{\infty}\left(1-\frac{s-\frac{1}{2}}{i t_{n}}\right) e^{s / s_{n}},
$$

where $\mu$ is defined in (46), $t_{n}$ run the roots of $\Xi(t)=0$, and the product runs over zeroes $s_{n}$ of (4);

\section{A5:}

$$
\xi(s)=\xi(0) \prod_{n=1}^{\infty}\left(1-\frac{s}{\widehat{s_{n}}}\right)\left(1-\frac{s}{1-\widehat{s_{n}}}\right),
$$

\section{A6:}

where the product runs over zeroes $s_{n}$ of (4);

$$
\xi(s)=\xi(1 / 2) \prod_{n=1}^{\infty}\left(1+\frac{(s-1 / 2)^{2}}{\tau_{n}^{2}}\right),
$$

where $\tau_{n}$ run the positive roots of $\Xi(t)=0$;

\section{A7:}

$$
\xi(s)=4 \int_{1}^{\infty} \frac{d\left(x^{\frac{3}{2}} \psi^{(1)}(x)\right)}{d x} x^{-\frac{1}{4}} \cosh \left(\frac{(s-1) \log x}{2}\right) d x .
$$

where $\psi(x)$ is defined in (8);

\section{A8:}

$$
\xi(s)=\sum_{k=0}^{\infty} \varphi_{k}\left(s-\frac{1}{2}\right)^{2 k}
$$

where $\varphi_{k}$ are expressed in (93).

Proof. A1 is the product of Hadamard [8]. A2 is the consequence of Theorem 1 and Conjecture 1. A3 is the product of Hadamard, which was proved in [8] and the book of Ingham ([47], p.57-58). A4 is derived from Theorem 2 and Conjecture 1. A5 is the product of Patterson ([17], p.34). A6 is deduced from Theorem 3 and Conjecture 1. $A 7$ is the Riemann integral representation theorem [2]. A8 is the Edwards series theorem, where $\varphi_{k}$ are expressed in (93). 
RIEMANN HYPOTHESIS

Theorem 14. Let

$$
N(T)=\#\{s=\sigma+i t: 0 \leq t \leq T, 0<\sigma<1, \xi(s)=0\}
$$

and

$$
N_{0}(T)=\#\{s=1 / 2+i t: 0 \leq t \leq T, \xi(s)=0\} .
$$

Then

$$
N(T)=N_{0}(T) .
$$

Proof. With Theorem 13, we give

$$
\xi\left(s_{n}\right)=\xi(1 / 2) \prod_{n=1}^{\infty}\left(1+\frac{\left(s_{n}-1 / 2\right)^{2}}{\tau_{n}^{2}}\right)=0,
$$

and we show

$$
s_{n}=1 / 2 \pm i \tau_{n},
$$

where $n \in \mathbb{N}$ and $\tau_{n}$ run the positive roots of $\Xi(t)=0$.

Thus, the required result follows.

The zeros (nontrivial zeros) for the entire Riemann zeta-function $\xi(s)$ are showed in Fig. 1.

Theorem 15. (Equivalence theorem II)

Let $s \in \mathbb{C} \backslash\{0,1\}$ and $n \in \mathbb{N}$. Then the following representations are equivalent:

B1:

$$
\varsigma(s)=\frac{1}{s(s-1)} \prod_{n=1}^{\infty}\left(1-\frac{s}{s_{n}}\right),
$$

where the product runs over zeroes $s_{n}$ of (4);

\section{B2:}

$$
\varsigma(s)=\frac{2 \xi(1 / 2)}{s(s-1)} \prod_{n=1}^{\infty}\left(1+\frac{i(s-1 / 2)}{t_{n}}\right),
$$

where $t_{n}$ run the roots of $\Xi(t)=0$;

\section{B3:}

$$
\varsigma(s)=\frac{1}{s(s-1)} e^{s \mu} \prod_{n=1}^{\infty}\left(1-\frac{s}{s_{n}}\right) e^{s / s_{n}},
$$

where $\mu$ is defined in (46) and the product runs over zeroes $s_{n}$ of (4); 


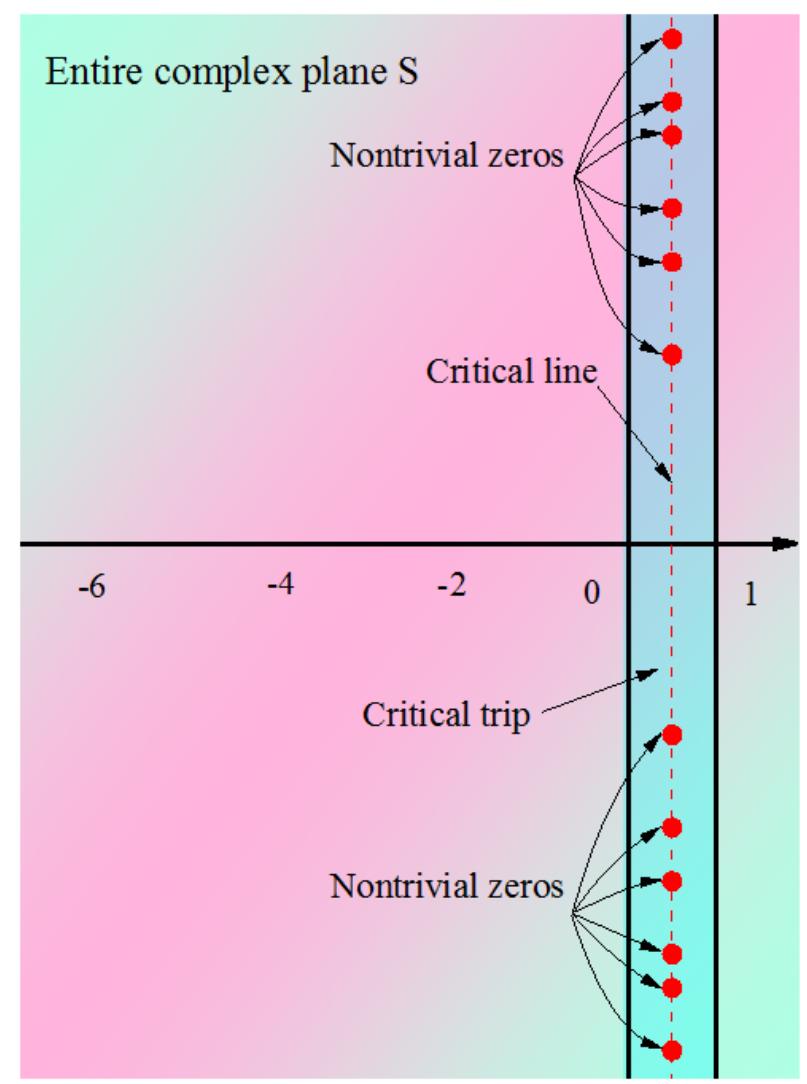

Figure 1. The distribution of the zeros (nontrivial zeros) for the entire Riemann zeta-function $\xi(s)$. The red points are represented as the nontrivial zeros for $\xi(s)$. All nontrivial zeros for $\xi(s)$ lie on the critical line $\operatorname{Re}(s)=1 / 2$ and in the critical strip $0<\operatorname{Re}(s)<1$.

\section{B4:}

$$
\varsigma(s)=\frac{2 \xi(1 / 2)}{s(s-1)} e^{s \mu} \prod_{n=1}^{\infty}\left(1-\frac{s-\frac{1}{2}}{i t_{n}}\right) e^{s / s_{n}},
$$

where $\mu$ is defined in (46), $t_{n}$ run the roots of $\Xi(t)=0$, and the product runs over zeroes $s_{n}$ of (4);

\section{B5:}

$$
\varsigma(s)=\frac{1}{s(s-1)} \prod_{n=1}^{\infty}\left(1-\frac{s}{\widehat{s_{n}}}\right)\left(1-\frac{s}{1-\widehat{s_{n}}}\right),
$$

where the product runs over zeroes $s_{n}$ of (4); 
B6:

$$
\varsigma(s)=\frac{2 \xi(1 / 2)}{s(s-1)} \prod_{n=1}^{\infty}\left(1+\frac{(s-1 / 2)^{2}}{\tau_{n}^{2}}\right),
$$

where $\tau_{n}$ run the positive roots of $\Xi(t)=0$;

\section{B7:}

$$
\varsigma(s)=\frac{8}{s(s-1)} \int_{1}^{\infty} \frac{d\left(x^{\frac{3}{2}} \psi^{(1)}(x)\right)}{d x} x^{-\frac{1}{4}} \cosh \left(\frac{(s-1) \log x}{2}\right) d x,
$$

\section{B8:}

$$
\varsigma(s)=\frac{1}{s(s-1)} \sum_{k=0}^{\infty} \varphi_{k}\left(s-\frac{1}{2}\right)^{2 k}
$$

where $\varphi_{k}$ are expressed in (93).

Proof. By (3) and (4), we arrive at

$$
\xi(s)=\frac{1}{2} s(s-1) \varsigma(s)
$$

which implies that

$$
\varsigma(s)=\frac{2 \xi(s)}{s(s-1)} .
$$

Thus, combining (161) and Theorem 13, we obtain the required results since $\xi(0)=$ $1 / 2$ ([46], p.49).

\section{Theorem 16. Let}

$$
\widehat{N}(T)=\#\{s=\sigma+i t: 0 \leq t \leq T, 0<\sigma<1, \varsigma(s)=0\}
$$

and

$$
\widehat{N}_{0}(T)=\#\{s=1 / 2+i t: 0 \leq t \leq T, \varsigma(s)=0\} .
$$

Then

$$
\widetilde{N}(T)=\widetilde{N}_{0}(T)
$$

Proof. By Theorem 15, we show

$$
\varsigma\left(s_{n}\right)=\frac{2 \xi(1 / 2)}{s_{n}\left(s_{n}-1\right)} \prod_{n=1}^{\infty}\left(1+\frac{i\left(s_{n}-1 / 2\right)}{t_{n}}\right)=0,
$$




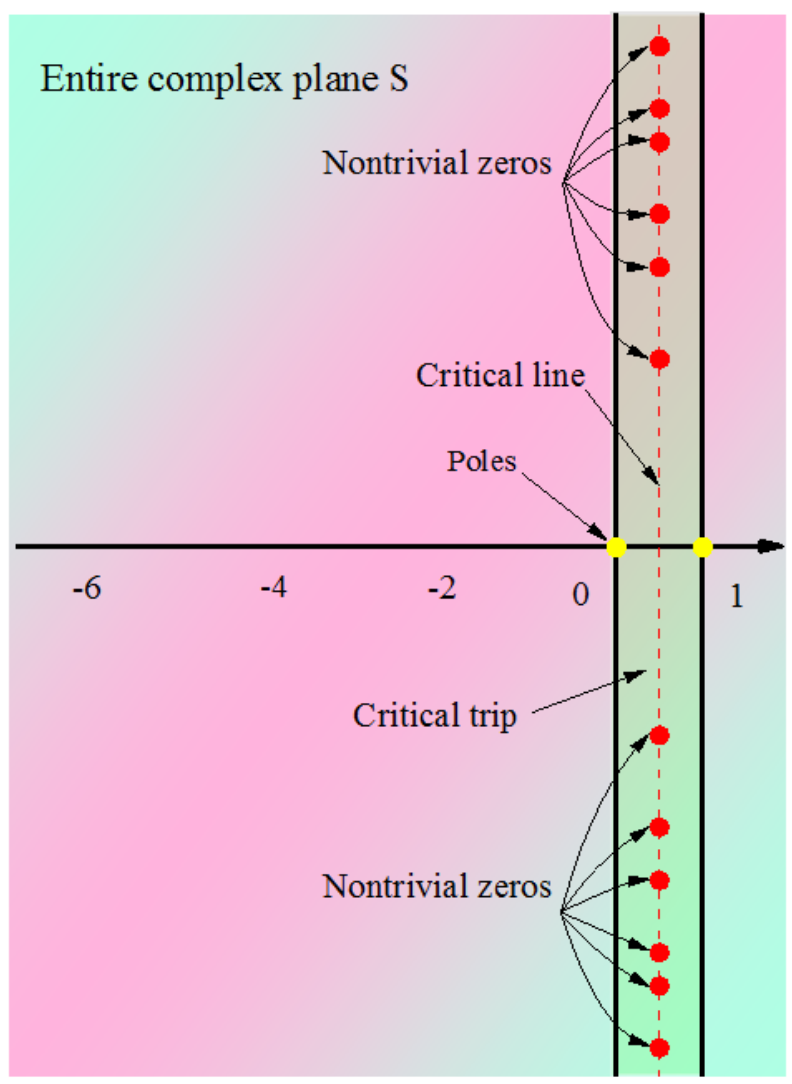

FiguRE 2. The distribution of the nontrivial zeros and poles for the completed Riemann zeta-function $\varsigma(s)$. The red points are represented as the nontrivial zeros for $\varsigma(s)$. The yellow points are represented as simple poles for $\varsigma(s)$. All nontrivial zeros for $\varsigma(s)$ lie on the critical line $\operatorname{Re}(s)=1 / 2$ and in the critical strip $0<\operatorname{Re}(s)<1$.

since $s_{n}\left(s_{n}-1\right) \neq 0$, and we obtain

$$
s_{n}=1 / 2+i t_{n},
$$

where $n \in \mathbb{N}$ and $t_{n}$ run the roots of $\Xi(t)=0$.

Thus, we finish the proof.

The nontrivial zeros for the completed Riemann zeta-function $\varsigma(s)$ and its poles are showed in Fig. 2.

Theorem 17. (Equivalence theorem III)

Let $s \in \mathbb{C} \backslash\{1\}$ and $n \in \mathbb{N}$. If $\Lambda(s)=2 \pi^{s / 2} /(s-1) \Gamma(s / 2+1)$, then the following representations are equivalent: 
C1:

$$
\zeta(s)=\xi(0) \Lambda(s) \prod_{n=1}^{\infty}\left(1-\frac{s}{s_{n}}\right),
$$

C2:

where the product runs over zeroes $s_{n}$ of (4);

$$
\zeta(s)=\xi(1 / 2) \Lambda(s) \prod_{n=1}^{\infty}\left(1+\frac{i(s-1 / 2)}{t_{n}}\right),
$$

where $t_{n}$ run the roots of $\Xi(t)=0$;

\section{C3:}

$$
\zeta(s)=\xi(0) \Lambda(s) e^{s \mu} \prod_{n=1}^{\infty}\left(1-\frac{s}{s_{n}}\right) e^{s / s_{n}},
$$

where $\mu$ is defined in (46) and the product runs over zeroes $s_{n}$ of (4); C4:

$$
\zeta(s)=\xi\left(\frac{1}{2}\right) \Lambda(s) e^{s \mu} \prod_{n=1}^{\infty}\left(1-\frac{s-\frac{1}{2}}{i t_{n}}\right) e^{s / s_{n}},
$$

where $\mu$ is defined in (46), $t_{n}$ run the roots of $\Xi(t)=0$, and the product runs over zeroes $s_{n}$ of (4);

\section{C5:}

$$
\zeta(s)=\xi(0) \Lambda(s) \prod_{n=1}^{\infty}\left(1-\frac{s}{\widehat{s_{n}}}\right)\left(1-\frac{s}{1-\widehat{s_{n}}}\right),
$$

\section{C6:}

where the product runs over zeroes $s_{n}$ of (4);

$$
\zeta(s)=\xi(1 / 2) \Lambda(s) \prod_{n=1}^{\infty}\left(1+\frac{(s-1 / 2)^{2}}{\tau_{n}^{2}}\right),
$$

where $\tau_{n}$ run the positive roots of $\Xi(t)=0$;

\section{C7:}

$$
\zeta(s)=4 \Lambda(s) \int_{1}^{\infty} \frac{d\left(x^{\frac{3}{2}} \psi^{(1)}(x)\right)}{d x} x^{-\frac{1}{4}} \cosh \left(\frac{(s-1) \log x}{2}\right) d x
$$

where $\psi(x)$ is defined in (8); 
C8:

$$
\zeta(s)=\Lambda(s) \sum_{k=0}^{\infty} \varphi_{k}\left(s-\frac{1}{2}\right)^{2 k}
$$

where $\varphi_{k}$ are expressed in (93).

Proof. With (4) we present

$$
\xi(s)=\frac{1}{2} s(s-1) \pi^{-s / 2} \Gamma(s / 2) \zeta(s),
$$

which leads to

$$
\zeta(s)=\Lambda(s) \xi(s)
$$

where

$$
\Lambda(s)=2 \pi^{s / 2} /(s-1) \Gamma(s / 2+1) .
$$

Thus, by (176) and Theorem 13, we get the desired results.

Remark. In the Landau's book ([6], p.313), he suggested that

$$
\zeta(s)=\frac{e^{s \ell}}{2(s-1) \Gamma(s / 2+1)} \prod_{n=1}^{\infty}\left(1-\frac{s}{s_{n}}\right) e^{s / s_{n}},
$$

where $s_{n}$ run the nontrivial zeros of $\zeta(s)=0$ and $\ell$ is a constant.

In his paper ([6], p.199), Landau proved that

$$
\zeta(s)=\frac{\pi^{s / 2} \Xi(0)}{(s-1) \Gamma(1+s / 2)} \prod_{n=1}^{\infty}\left(1+\frac{\left(s-\frac{1}{2}\right)^{2}}{\tau_{n}^{2}}\right),
$$

where $\tau_{n}$ run the positive roots of $\Xi(t)=0$.

Here, we have to point out that $C 3$ and $C 6$ are equivalent to (178) and (179), respectively.

\section{Theorem 18. Let}

$$
\widetilde{N}(T)=\#\{s=\sigma+i t: 0 \leq t \leq T, 0<\sigma<1, \zeta(s)=0\}
$$

and

$$
\widetilde{N}_{0}(T)=\#\{s=1 / 2+i t: 0 \leq t \leq T, \zeta(s)=0\}
$$

Then

$$
\widetilde{N}(T)=\widetilde{N}_{0}(T)
$$




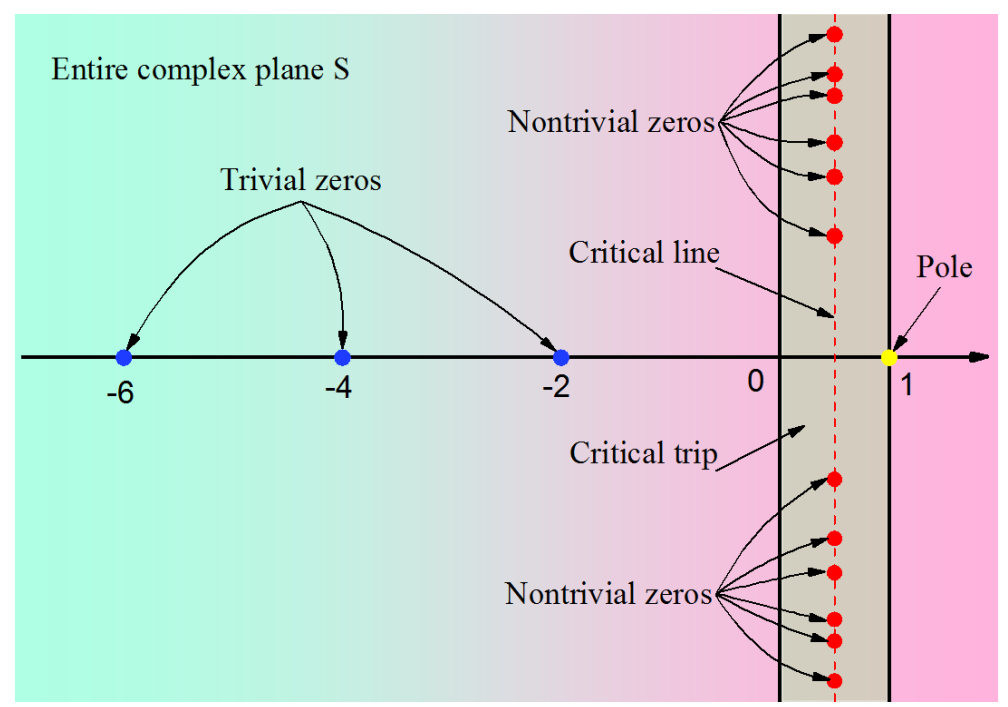

FIGURE 3. The distribution of the trivial, pole, and nontrivial zeros for the Riemann zeta-function $\zeta(s)$. The red points are represented as the nontrivial zeros for $\zeta(s)$. The blue points are represented as the trivial zeros for $\zeta(s)$. The yellow point is represented as a simple pole for $\zeta(s)$. All nontrivial zeros for $\zeta(s)$ lie on the critical line $\operatorname{Re}(s)=1 / 2$ and in the critical strip $0<\operatorname{Re}(s)<1$.

Proof. Making use of Theorem 17, we give

$$
\begin{aligned}
& \zeta\left(s_{n}\right) \\
& =\xi(1 / 2) \Lambda\left(s_{n}\right) e^{\mu s_{n}} \prod_{n=1}^{\infty}\left(1-\frac{s_{n}-\frac{1}{2}}{i t_{n}}\right) e^{s_{n} / s_{n}} \\
& =\xi(1 / 2) \Lambda\left(s_{n}\right) e^{\mu s_{n}} \prod_{n=1}^{\infty}\left(1-\frac{s_{n}-\frac{1}{2}}{i t_{n}}\right) \\
& =0
\end{aligned}
$$

since

$$
\Lambda\left(s_{n}\right)=2 \pi^{s_{n} / 2} /\left(s_{n}-1\right) \Gamma\left(s_{n} / 2+1\right) \neq 0
$$

and we have

$$
s_{n}=1 / 2+i t_{n}
$$

where $n \in \mathbb{N}$ and $t_{n}$ run the roots of $\Xi(t)=0$.

Thus, the proof is completed.

The trivial, pole, and nontrivial zeros for the Riemann zeta-function $\zeta(s)$ are showed in Fig. 3. 
Remark. For the history of Theorems 14, 16 and 18, see [22, 23].

By applying the theory of the entire function ([51], p.29), we show that the canonical product

$$
\prod_{n=1}^{\infty} \mathbb{E}\left(t / t_{n}, 0\right)=\Xi(0) \prod_{n=1}^{\infty}\left(1-\frac{t^{2}}{\tau_{n}^{2}}\right)=\prod_{n=1}^{\infty}\left(1-\frac{t}{t_{n}}\right)=\frac{\Xi(t)}{\Xi(0)},
$$

is of growth $(1,0)$ since $\sum_{n=1}^{\infty} t_{n}^{-2}$ converges.

In a similar manner, by theory of the entire function $([51]$, p.29), the canonical product

$$
\prod_{n=1}^{\infty} \mathbb{E}\left(s / s_{n}, 0\right)=\prod_{n=1}^{\infty}\left(1-\frac{s}{s_{n}}\right)=\frac{\xi(s)}{\xi(0)}
$$

is also of growth $(1,0)$ since $\sum_{n=1}^{\infty} s_{n}^{-2}$ converges.

Heath-Brown in 2005 [36] pointed out that all zeros for $\xi(s)$ are simple. We now deduce it is true since by $(140)$, we find that all zeros for $\xi(s)$ are different and that $\xi(s)$ is an entire function.

\section{REFERENCES}

[1] Euler, L. (1744). Variae observationes circa series infinitas. Commentarii academiae scientiarum Petropolitanae, 9, 160-188.

[2] Riemann, B. (1859.). Über die Anzahl der Primzahlen unter einer gegebenen Grösse. Monatsberichte der Deutschen Akademie der Wissenschaften zu Berlin, 2, 671-680.

[3] Edwards, H. M. (1974). Riemann's zeta function. Academic press, New York.

[4] Yang, X. J. (2021). An introduction to hypergeometric, supertrigonometric, and superhyperbolic functions. Academic Press, New York.

[5] Neukirch, J. (1994). Algebraic number theory. Springer, New York.

[6] Landau, E. (1909). Handbuch der Lehre von der Verteilung der Primzahlen, Teubner, Leipzig.

[7] Pólya, G. (1927). Über die algebraisch-funktionentheoretischen untersuchungen von J. L. W. V. Jensen. Mathematisk-Fysiske Meddelelsev, 17(4), 1-33.

[8] Hadamard, J. (1893). Étude sur les propriétés des fonctions entières et en particulier d'une fonction considérée par Riemann. Journal de mathématiques pures et appliquées, 9, 171-216.

[9] Hardy, G. H. (1914). Sur les zéros de la fonction $\zeta(s)$ de Riemann. Comptes Rendus de l'Académie des Sciences de Paris, 158, 1012-1014.

[10] Hardy, G. H., Littlewood, J. E. (1921). The zeros of Riemann's zeta-function on the critical line. Mathematische Zeitschrift, 10(3), 283-317.

[11] Csordas, G., Norfolk, T. S., Varga, R. S. (1986). The Riemann hypothesis and the Turán inequalities. Transactions of the American Mathematical Society, 296(2), 521-541.

[12] Conrey, J. B., Ghosh, A. (1994). Turán inequalities and zeros of Dirichlet series associated with certain cusp forms. Transactions of the American Mathematical Society, 342(1), 407-419. 
[13] Pólya, G., Schur, J. (1914). Über zwei Arten von Faktorenfolgen in der Theorie der algebraischen Gleichungen. Journal für die reine und angewandte Mathematik, 144, 89-113.

[14] Dimitrov, D. K., Cheikh, Y. B. (2009). Laguerre polynomials as Jensen polynomials of Laguerre-Pólya entire functions. Journal of Computational and Applied Mathematics, 233(3), 703-707.

[15] Cahen, E. (1894). Sur la fonction $\zeta(s)$ de Riemann et sur des fonctions analogues. Annales scientifiques de l'École Normale Supérieure, 11, 75-164.

[16] Titchmarsh, E. C. (1927). A consequence of the Riemann hypothesis. Journal of the London Mathematical Society, 1(4), 247-254.

[17] Patterson, S. J. (1995). An introduction to the theory of the Riemann zeta-function. Cambridge University Press, Cambridge.

[18] Landau, E. (1908). Beiträge zur analytischen zahlentheorie. Rendiconti Del Circolo Matematico Di Palermo, 26(1), 169-302.

[19] Euler, L. (1768). Remarques sur un beau rapport entre les series des puissances tant directes que reciproques. Memoires de l'academie des sciences de Berlin, 17, 83-106.

[20] Hadamard, J. (1896). Sur la distribution des zéros de la fonction $\zeta(s)$ et ses conséquences arithmétiques. Bulletin de la Societé mathematique de France, 24, 199-220.

[21] Vallée-Poussin, C. D. L. de la. (1896). Recherches analytiques sur la théorie des nombres premiers, I-III. Annales de la Société scientifique de Bruxelles, 20, 183-256.

[22] Iwaniec, H. (2014). Lectures on the Riemann zeta function. American Mathematical Society, New York.

[23] Ivic, A. (2012). The Riemann Zeta-function: Theory and Applications, Courier Corporation.

[24] Landau, E. (1915). Über die Hardysche Entdeckung unendlich vieler Nullstellen der Zetafunktion mit reellem Teil 1/2. Mathematische Annalen, 76(2-3), 212-243.

[25] Heath-Brown, D. R. (1979). Simple zeros of the Riemann zeta-function on the critical line. Bulletin of the London Mathematical Society, 11(1), 17-18.

[26] Mangoldt, H. V. (1905). Zur Verteilung der Nullstellen der Riemannschen Funktion $\xi(t)$. Mathematische Annalen, 60(1), 1-19.

[27] Siegel, C. L. (1932). Über Riemann's Nachlass zur analytischen Zahlentheorie. Quellen und Studien zur Geschichte der Mathematik, Astronomie und Physik, 2, 45-80.

[28] Siegel, C. L. (1943). Contributions to the theory of the Dirichlet $L$-series and the Epstein zeta-functions. Annals of Mathematics, 44(2), 143-172.

[29] Brent, R. P. (1979). On the zeros of the Riemann zeta function in the critical strip. Mathematics of Computation, 33(148), 1361-1361.

[30] Odlyzko, A. M., Schönhage, A. (1988). Fast algorithms for multiple evaluations of the Riemann zeta function. Transactions of the American Mathematical Society, 309(2), 797-809.

[31] Chen, W., Jia, D., Wang, L. (2019). Higher order Turán inequalities for the partition function. Transactions of the American Mathematical Society, 372(3), 2143-2165.

[32] Euler, L. (1796). Introduction à l'analyse infinitésimale. Chez Barrois, l'an IV-V.

[33] Hilbert, D. (1902). Mathematische probleme. Bulletin of the American Mathematical Society, 1902, 8(11), 437-479.

[34] Sarnak, P. (2004). Problems of the Millenium: The Riemann Hypothesis, Clay Mathematics Institute, New York.

[35] Bombieri, E. (2019). New progress on the zeta function: from old conjectures to a major breakthrough. Proceedings of the National Academy of Sciences, 116(23), 11085-11086. 
[36] Heath-Brown, D. (2005). Prime number theory and the Riemann zeta-function. In: F. Mezzadri \& N. Snaith (Eds.), Recent Perspectives in Random Matrix Theory and Number Theory (London Mathematical Society Lecture Note Series, pp. 1-30). Cambridge: Cambridge University Press.

[37] Conrey, B. (2015). Riemann's Hypothesis. In: L.-Z. Ji, F. Oort, S.-T. Yau (Eds.), The Legacy of Bernhard Riemann After One Hundred and Fifty Years (Advanced Lectures in Mathematics 35, p.107-190). Higher Education Press/International Press.

[38] Montgomery, H. L. (1973). The pair correlation of zeros of the zeta function. Proceedings of Symposia in Pure Mathematics, 24, 181-193.

[39] Chirre, A., Gonçalves, F., de Laat, D. (2020). Pair correlation estimates for the zeros of the zeta function via semidefinite programming. Advances in Mathematics, 361, 106926.

[40] Berry, M. V., Keating, J. P. (1999). The Riemann zeros and eigenvalue asymptotics. SIAM review, 41(2), 236-266.

[41] Bender, C. M., Brody, D. C., Müller, M. P. (2017). Hamiltonian for the zeros of the Riemann zeta function. Physical Review Letters, 118(13), 130201.

[42] Yang, C. N., Lee, T. D. (1952). Statistical theory of equations of state and phase transitions. I. Theory of condensation. Physical Review, 87(3), 404.

[43] Kim, S. Y. (2004). Yang-Lee zeros of the antiferromagnetic Ising model. Physical Review Letters, 93(13), 130604.

[44] Borwein, P. B., Borwein, P., Choi, S., Rooney, B., Weirathmueller, A. (Eds.). (2008). The Riemann hypothesis: a resource for the afficionado and virtuoso alike. Springer.

[45] Griffin, M., Ono, K., Rolen, L., Zagier, D. (2019). Jensen polynomials for the Riemann zeta function and other sequences. Proceedings of the National Academy of Sciences, 116 (24), 11103-11110.

[46] Broughan, K. (2017). Equivalents of the Riemann Hypothesis: Volume 2, Analytic Equivalents. Cambridge University Press.

[47] Ingham, A. E. (1964). The distribution of prime numbers. Cambridge University Press, London.

[48] Titchmarsh, E. C. (1930). The zeta-function of Riemann, The University Press, Cambridge.

[49] Titchmarsh, E. C., Heath-Brown, D. R.(1986). The theory of the Riemann zeta-function. Oxford University Press.

[50] Levin, B. Y. (1996). Lectures on entire functions. American Mathematical Society, New York.

[51] Boas, R. P. (1954), Entire Functions, Academic Press, New York.

[52] Jensen, J. L. (1913). Recherches sur la théorie des équations. Acta Mathematica, 36(1), 181195.

[53] Lagarias, J. C., Montague, D. (2011). The integral of the Riemann xi-function. Commentarii mathematici Universitatis Sancti Pauli, 60(1-2), 143-169.

[54] Wintner, A. (1935). A note on the Riemann $\xi$-function. Journal of the London Mathematical Society, 1(2), 82-83.

[55] Dixit, A., Robles, N., Roy, A., Zaharescu, A. (2015). Zeros of combinations of the Riemann $\xi$-function on bounded vertical shifts. Journal of Number Theory, 149, 404-434.

E-mail address: dyangxiaojun@163.com; xjyang@cumt.edu.cn

School of Mathematics, China University of Mining and Technology, Xuzhou 221116, CHINA 